\title{
Jacquet Modules of Principal Series Generated by the Trivial $K$-Type
}

\author{
By
}

Noriyuki ABE*

\begin{abstract}
We propose a new approach to the study of the Jacquet module of a HarishChandra module of a real semisimple Lie group. Using this method, we investigate the structure of the Jacquet module of a principal series representation generated by the trivial $K$-type.
\end{abstract}

\section{$\S 1$. Introduction}

Let $G$ be a real semisimple Lie group. By Casselman's subrepresentation theorem, any irreducible admissible representation $\mathbb{X}$ is realized as a subrepresentation of a certain non-unitary principal series representation. Such an embedding is a powerful tool to study an irreducible admissible representation but the subrepresentation theorem dose not tell us how it can be realized.

Casselman [Cas80] introduced the Jacquet module $J(\mathbb{X})$ of $\mathbb{X}$. This important object retains all information of embedding given by the subrepresentation theorem. For example, Casselman's subrepresentation theorem is equivalent to $J(\mathbb{X}) \neq 0$. However the structure of $J(\mathbb{X})$ is very intricate and difficult to determine. We remark that if $G$ has real rank one, then Collingwood [Col85] has computed the detailed structure of Jacquet modules.

In this paper we give generators of the Jacquet module of a principal series representation generated by the trivial $K$-type. This representation is

\footnotetext{
Communicated by M. Kashiwara. Received December 17, 2007. Revised April 18, 2008, May 8, 2008.

2000 Mathematics Subject Classification(s): 22E47.

* Graduate School of Mathematical Sciences, the University of Tokyo, 3-8-1 Komaba, Meguro-ku, Tokyo 153-8914, Japan.

e-mail: abenori@ms.u-tokyo.ac.jp
}

(C) 2008 Research Institute for Mathematical Sciences, Kyoto University. All rights reserved. 
deeply related to the harmonic analysis on the Riemannian symmetric space $G / K\left[\mathrm{Hel} 62, \mathrm{KKM}^{+} 78\right]$. Let $\mathbb{Z}$ be the ring of integers, $\mathfrak{g}_{0}$ the Lie algebra of $G$, $\theta$ a Cartan involution of $\mathfrak{g}_{0}, \mathfrak{g}_{0}=\mathfrak{k}_{0} \oplus \mathfrak{a}_{0} \oplus \mathfrak{n}_{0}$ the Iwasawa decomposition of $\mathfrak{g}_{0}, \mathfrak{m}_{0}$ the centralizer of $\mathfrak{a}_{0}$ in $\mathfrak{k}_{0}, W$ the little Weyl group for $\left(\mathfrak{g}_{0}, \mathfrak{a}_{0}\right), e \in W$ the unit element of $W, \Sigma$ the restricted root system for $\left(\mathfrak{g}_{0}, \mathfrak{a}_{0}\right), \mathfrak{g}_{0, \alpha}$ the root space for $\alpha \in \Sigma, \Sigma^{+}$the positive system of $\Sigma$ such that $\mathfrak{n}_{0}=\bigoplus_{\alpha \in \Sigma^{+}} \mathfrak{g}_{0, \alpha}$, $\rho=\sum_{\alpha \in \Sigma^{+}}\left(\operatorname{dim} \mathfrak{g}_{0, \alpha} / 2\right) \alpha, \mathcal{P}=\left\{\sum_{\alpha \in \Sigma^{+}} n_{\alpha} \alpha \mid n_{\alpha} \in \mathbb{Z}\right\}, \mathcal{P}^{+}=\left\{\sum_{\alpha \in \Sigma^{+}} n_{\alpha} \alpha \mid\right.$ $\left.n_{\alpha} \in \mathbb{Z}, n_{\alpha} \geq 0\right\}$. For a Lie algebra $\mathfrak{h}$, let $U(\mathfrak{h})$ be the universal enveloping algebra of $\mathfrak{h}$.

For a Harish-Chandra module $\mathbb{X}$, the Jacquet module $J(\mathbb{X})$ of $\mathbb{X}$ is defined by

$$
J(\mathbb{X})=\left\{x \in \underset{k}{\lim } \mathbb{X} / \mathfrak{n}_{0}^{k} \mathbb{X} \mid \operatorname{dim} U\left(\mathfrak{a}_{0}\right) x<\infty\right\} .
$$

In this paper we prove the following theorem.

Theorem 1.1 (Theorem 4.10, Proposition 5.1). Let $\lambda \in \operatorname{Hom}_{\mathbb{R}}\left(\mathfrak{a}_{0}, \mathbb{C}\right)$ and $I(\lambda)$ be the unique principal series representation with an infinitesimal character $\lambda$ generated by the trivial $K$-type. Assume that $\lambda$ is regular. Set $\mathcal{W}(w)=\left\{w^{\prime} \in W \mid w \lambda-w^{\prime} \lambda \in 2 \mathcal{P}^{+}\right\}$for $w \in W$. Then there exist generators $\left\{v_{w} \mid w \in W\right\}$ of $J(I(\lambda))$ such that

$$
\left\{\begin{array}{l}
(H-(\rho+w \lambda)(H)) v_{w} \in \sum_{w^{\prime} \in \mathcal{W}(w)} U(\mathfrak{g}) v_{w^{\prime}} \text { for all } H \in \mathfrak{a}_{0}, \\
X v_{w} \in \sum_{w^{\prime} \in \mathcal{W}(w)} U(\mathfrak{g}) v_{w^{\prime}} \text { for all } X \in \mathfrak{m}_{0} \oplus \theta\left(\mathfrak{n}_{0}\right) .
\end{array}\right.
$$

Hence $v_{w}$ is a lowest weight vector of $J(I(\lambda)) / \sum_{w^{\prime} \in \mathcal{W}(w)} U(\mathfrak{g}) v_{w^{\prime}}$.

Recall the definition of generalized Verma modules. For $\mu \in \operatorname{Hom}_{\mathbb{R}}\left(\mathfrak{a}_{0}, \mathbb{C}\right)$, let $\mathbb{C}_{\mu}$ be the one-dimensional representation of $\mathfrak{m}_{0} \oplus \mathfrak{a}_{0} \oplus \theta\left(\mathfrak{n}_{0}\right)$ defined by $\mathfrak{m}_{0} \oplus \mathfrak{a}_{0} \oplus \theta\left(\mathfrak{n}_{0}\right) \rightarrow \mathfrak{a}_{0} \rightarrow \mathbb{C}$, where the first map is the projection to the direct summand and the second map is $\mu$. Then the generalized Verma module $M(\mu)$ is defined by $M(\mu)=U(\mathfrak{g}) \otimes_{U\left(\mathfrak{m}_{0} \oplus \mathfrak{a}_{0} \oplus \theta\left(\mathfrak{n}_{0}\right)\right)} \mathbb{C}_{\mu+\rho}$.

We enumerate $W=\left\{w_{1}, w_{2}, \ldots, w_{r}\right\}$ in such a way that $\operatorname{Re} w_{1} \lambda \geq \operatorname{Re} w_{2} \lambda \geq$ $\cdots \geq \operatorname{Re} w_{r} \lambda$. Set $V_{i}=\sum_{j \geq i} U(\mathfrak{g}) v_{w_{j}}$. Then by Theorem 1.1 we have a surjective map $M\left(w_{i} \lambda\right) \rightarrow V_{i} / V_{i+1}$ where $M\left(w_{i} \lambda\right)$ is the generalized Verma module. This map is isomorphic. Namely we can prove the following theorem.

Theorem 1.2 (Theorem 5.5). There exists a filtration $J(I(\lambda))=V_{1} \supset$ $V_{2} \supset \cdots \supset V_{r+1}=0$ of $J(I(\lambda))$ such that $V_{i} / V_{i+1} \simeq M\left(w_{i} \lambda\right)$. Moreover if $w \lambda-\lambda \notin 2 \mathcal{P}$ for $w \in W \backslash\{e\}$ then $J(I(\lambda)) \simeq \bigoplus_{w \in W} M(w \lambda)$.

This theorem does not need the assumption that $\lambda$ is regular. If $G$ is split and $I(\lambda)$ is irreducible, Collingwood [Col91] proved Theorem 1.2. 
For example, we obtain the following for $\mathfrak{g}_{0}=\mathfrak{s l}(2, \mathbb{R})$ : Choose a basis $\left\{H, E_{+}, E_{-}\right\}$of $\mathfrak{g}_{0}$ such that $\mathbb{R} H=\mathfrak{a}_{0}, \mathbb{R} E_{+}=\mathfrak{n}_{0},\left[H, E_{ \pm}\right]= \pm E_{ \pm}$and $\theta\left(E_{+}\right)=$ $-E_{-}$. Then $\Sigma^{+}=\{\alpha\}$ where $\alpha(H)=1$. Let $\lambda=r \alpha$ for $r \in \mathbb{C}$. We may assume $\operatorname{Re} r \geq 0$. By Theorems 1.1 and 1.2, we have an exact sequence

$$
0 \longrightarrow M(-r \alpha) \longrightarrow J(I(r \alpha)) \longrightarrow M(r \alpha) \longrightarrow 0 .
$$

Consider the case where $\lambda$ is integral, i.e., $2 r \in \mathbb{Z}$. If $r \notin \mathbb{Z}$ then this sequence splits by Theorem 1.2. On the other hand, if $r \in \mathbb{Z}$ then by a direct calculation using the method introduced in this paper we can show it does not split. Notice that $I(r \alpha)$ is irreducible if and only if $r \in \mathbb{Z}$. Then we have the following; if $\lambda$ is integral then $J(I(\lambda))$ is isomorphic to the direct sum of generalized Verma modules if and only if $I(\lambda)$ is reducible.

We summarize the content of this paper. In Section 2, we prove our main theorem for the case $G=S L(2, \mathbb{R})$. We do not need this section later, but it serves as a prototype for the arguments that follow. We begin to treat the general case from Section 3 on. In Section 3 we show fundamental properties of Jacquet modules and introduce a certain extension of the universal enveloping algebra. We construct special elements in the Jacquet module in Section 4. In Section 5 we prove our main theorem in the case of a regular infinitesimal character using the result of Section 4. We complete the proof in Section 6 using the translation principle.

\section{Notation}

Throughout this paper we use the following notation. As usual we denote the ring of integers, the set of non-negative integers, the set of positive integers, the real number field and the complex number field by $\mathbb{Z}, \mathbb{Z}_{\geq 0}, \mathbb{Z}_{>0}, \mathbb{R}$ and $\mathbb{C}$ respectively. Let $\mathfrak{g}_{0}$ be a real semisimple Lie algebra. Fix a Cartan involution $\theta$ of $\mathfrak{g}_{0}$. Let $\mathfrak{g}_{0}=\mathfrak{k}_{0} \oplus \mathfrak{s}_{0}$ be the decomposition of $\mathfrak{g}_{0}$ into the +1 and -1 eigenspaces for $\theta$. Take a maximal abelian subspace $\mathfrak{a}_{0}$ of $\mathfrak{s}_{0}$ and let $\mathfrak{g}_{0}=$ $\mathfrak{k}_{0} \oplus \mathfrak{a}_{0} \oplus \mathfrak{n}_{0}$ be the corresponding Iwasawa decomposition of $\mathfrak{g}_{0}$. Set $\mathfrak{m}_{0}=$ $\left\{X \in \mathfrak{k}_{0} \mid[H, X]=0\right.$ for all $\left.H \in \mathfrak{a}_{0}\right\}$. Then $\mathfrak{p}_{0}=\mathfrak{m}_{0} \oplus \mathfrak{a}_{0} \oplus \mathfrak{n}_{0}$ is a minimal parabolic subalgebra of $\mathfrak{g}_{0}$. Write $\mathfrak{g}$ for the complexification of $\mathfrak{g}_{0}$ and $U(\mathfrak{g})$ for the universal enveloping algebra of $\mathfrak{g}$. We use analogous notation for other Lie algebras.

Set $\mathfrak{a}^{*}=\operatorname{Hom}_{\mathbb{C}}(\mathfrak{a}, \mathbb{C})$ and $\mathfrak{a}_{0}^{*}=\operatorname{Hom}_{\mathbb{R}}\left(\mathfrak{a}_{0}, \mathbb{R}\right)$. Let $\Sigma \subset \mathfrak{a}^{*}$ be the restricted root system for $(\mathfrak{g}, \mathfrak{a})$ and $\mathfrak{g}_{\alpha}$ the root space for $\alpha \in \Sigma$. Let $\Sigma^{+}$be the positive root system determined by $\mathfrak{n}$, i.e., $\mathfrak{n}=\bigoplus_{\alpha \in \Sigma^{+}} \mathfrak{g}_{\alpha} . \Sigma^{+}$determines the set of simple roots $\Pi=\left\{\alpha_{1}, \alpha_{2}, \ldots, \alpha_{l}\right\}$. We define a total order on $\mathfrak{a}_{0}^{*}$ by the following; for $c_{i}, d_{i} \in \mathbb{R}$ we define $\sum_{i} c_{i} \alpha_{i}>\sum_{i} d_{i} \alpha_{i}$ if and only if there exists an 
integer $k$ such that $c_{1}=d_{1}, \ldots, c_{k}=d_{k}$ and $c_{k+1}>d_{k+1}$. Let $\left\{H_{1}, H_{2}, \ldots, H_{l}\right\}$ be the dual basis of $\left\{\alpha_{i}\right\}$. Write $W$ for the little Weyl group for $\left(\mathfrak{g}_{0}, \mathfrak{a}_{0}\right)$ and $e$ for the unit element of $W$. Set $\mathcal{P}=\left\{\sum_{\alpha \in \Sigma^{+}} n_{\alpha} \alpha \mid n_{\alpha} \in \mathbb{Z}\right\}, \mathcal{P}^{+}=\left\{\sum_{\alpha \in \Sigma^{+}} n_{\alpha} \alpha \mid\right.$ $\left.n_{\alpha} \in \mathbb{Z}_{\geq 0}\right\}$ and $\mathcal{P}^{++}=\mathcal{P}^{+} \backslash\{0\}$. Let $m$ be the dimension of $\mathfrak{n}$. Fix a basis $E_{1}, E_{2}, \ldots, E_{m}$ of $\mathfrak{n}$ such that each $E_{i}$ is a restricted root vector. Let $\beta_{i}$ be a restricted root such that $E_{i} \in \mathfrak{g}_{\beta_{i}}$. For $\mathbf{n}=\left(\mathbf{n}_{i}\right) \in \mathbb{Z}_{\geq 0}^{m}$ we denote $E_{1}^{\mathbf{n}_{1}} E_{2}^{\mathbf{n}_{2}} \cdots E_{m}^{\mathbf{n}_{m}}$ by $E^{\mathbf{n}}$.

For $x=\left(x_{1}, x_{2}, \ldots, x_{n}\right) \in \mathbb{Z}_{\geq 0}^{n}$, we write $|x|=x_{1}+x_{2}+\cdots+x_{n}$ and $x !=x_{1} ! x_{2} ! \cdots x_{n} !$

For a $\mathbb{C}$-algebra $R$, let $M\left(r, r^{\prime}, R\right)$ be the space of $r \times r^{\prime}$ matrices with entries in $R$ and $M(r, R)=M(r, r, R)$. Write $1_{r} \in M(r, R)$ for the identity matrix.

\section{§2. The Case $S L(2, \mathbb{R})$}

Definition 2.1 (Jacquet module [Cas80]). $\quad$ Let $\mathbb{X}$ be a $U(\mathfrak{g})$-module. Define modules $\widehat{J}(\mathbb{X})$ and $J(\mathbb{X})$ by

$$
\begin{aligned}
& \widehat{J}(\mathbb{X})=\overleftarrow{l}_{k}^{\lim } \mathbb{X} / \mathfrak{n}^{k} \mathbb{X}, \\
& J(\mathbb{X})=\widehat{J}(\mathbb{X})_{\mathfrak{a} \text {-finite }}=\{x \in \widehat{J}(\mathbb{X}) \mid \operatorname{dim} U(\mathfrak{a}) x<\infty\} .
\end{aligned}
$$

We call $J(\mathbb{X})$ the Jacquet module of $\mathbb{X}$.

Remark 2.2. In some articles, e.g., Wallach [Wal88, 4.1.5], the definition of the Jacquet module is different from what we give here. These Jacquet modules are dual to each other (cf. Matumoto [Mat90, Corollary 4.7.4]).

In this section, let $G=S L(2, \mathbb{R})$.

Take a basis $H, E_{+}, E_{-}$of $\mathfrak{s l}(2, \mathbb{R})$ such that $\left[H, E_{ \pm}\right]= \pm E_{ \pm},\left[E_{+}, E_{-}\right]=$ $H, \mathfrak{a}_{0}=\mathbb{R} H, \mathfrak{n}_{0}=\mathbb{R} E_{+}$and $\theta\left(E_{+}\right)=-E_{-}$. Fix $\lambda \in \mathbb{C} \backslash\{0\}$ such that $\operatorname{Re} \lambda \geq 0$. Let $I(\lambda)$ be the unique principal series representation generated by the trivial $K$-type with an infinitesimal character $\lambda$. Then $I(\lambda)$ has a generator $u_{\lambda}$ and the following relations:

$$
\begin{aligned}
\left(H^{2}-H+2 E_{+} E_{-}\right) u_{\lambda} & =\left(\lambda^{2}-1 / 4\right) u_{\lambda}, \\
\left(E_{+}-E_{-}\right) u_{\lambda} & =0 .
\end{aligned}
$$

The first relation says that $I(\lambda)$ has an infinitesimal character $\lambda\left(\left(H^{2}-H+\right.\right.$ $\left.2 E_{+} E_{-}\right)$is the Casimir element of $\left.\mathfrak{s l}(2, \mathbb{C})\right)$ and the second relation says that $u_{\lambda}$ belongs to its trivial $K$-type. 
By the relations, we have $\left(H^{2}-H+2 E_{+}^{2}\right) u_{\lambda}=\left(\lambda^{2}-1 / 4\right) u_{\lambda}$. Put $\overline{u_{\lambda}}=$ $u_{\lambda}+\mathfrak{n} I(\lambda) \in I(\lambda) / \mathfrak{n} I(\lambda)$. Then we have $(H-(\lambda+1 / 2))(H-(-\lambda+1 / 2)) \overline{u_{\lambda}}=0$. Hence the dimension of $I(\lambda) / \mathfrak{n} I(\lambda)$ is 2 and the eigenvalues of $H$ are $\pm \lambda+1 / 2$.

Put $u_{1}=(H-(-\lambda+1 / 2)) u_{\lambda}, u_{2}=(H-(\lambda+1 / 2)) u_{\lambda}, u={ }^{t}\left(u_{1}, u_{2}\right)$. Then $\left\{u_{1}+\mathfrak{n} I(\lambda), u_{2}+\mathfrak{n} I(\lambda)\right\}$ is a basis of $I(\lambda) / \mathfrak{n} I(\lambda)$. We have

$$
\left(H 1_{2}-Q\right) u=\frac{1}{\lambda}\left(\begin{array}{ll}
-1 & 1 \\
-1 & 1
\end{array}\right) E_{+}^{2} u \quad \text { where } \quad Q=\left(\begin{array}{cc}
\lambda+1 / 2 & 0 \\
0 & -\lambda+1 / 2
\end{array}\right) .
$$

Put

$$
R=\frac{1}{\lambda}\left(\begin{array}{ll}
-1 & 1 \\
-1 & 1
\end{array}\right)
$$

Then we have $\left(H 1_{2}-Q-R E_{+}^{2}\right) u=0$. Define a $\mathbb{C}$-algebra $\widehat{\mathcal{E}}(\mathfrak{n})$ by $\widehat{\mathcal{E}}(\mathfrak{n})=$ $\varliminf_{k} U(\mathfrak{n}) / \mathfrak{n}^{k} U(\mathfrak{n})$. In this case, we have $\widehat{\mathcal{E}}(\mathfrak{n})=\mathbb{C}\left[\left[E_{+}\right]\right]$. This is a complete local ring with the maximal ideal $E_{+} \mathbb{C}\left[\left[E_{+}\right]\right]=\mathfrak{n} \widehat{\mathcal{E}}(\mathfrak{n})$. Notice that the $\mathbb{C}$ algebra $\widehat{\mathcal{E}}(\mathfrak{n})$ acts on $\widehat{J}(I(\lambda))$.

The crucial fact is the following lemma. The lemma says that the action of $H$ can be expressed by an upper triangular matrix.

Lemma 2.3. There exist $L \in 1_{2}+M(2, \mathfrak{n} \widehat{\mathcal{E}}(\mathfrak{n}))$ and $T \in M(2, U(\mathfrak{n}))$ which have the following properties.

(1) We have $\left(H 1_{2}-Q-R E_{+}^{2}\right) L=L\left(H 1_{2}-Q-T\right)$.

(2) If $\lambda \notin \mathbb{Z}_{>0}$, then $T=0$.

(3) If $\lambda \in \mathbb{Z}_{>0}$, then $T=T_{\lambda} E_{+}^{2 \lambda}$ where

$$
T_{\lambda}=\left(\begin{array}{ll}
0 & t \\
0 & 0
\end{array}\right)
$$

for some $t \in \mathbb{C}$.

Notice that $L$ is invertible since $\operatorname{det} L \in 1+\mathfrak{n} \widehat{\mathcal{E}}(\mathfrak{n})$ is invertible. (Recall that $\widehat{\mathcal{E}}(\mathfrak{n})$ is a local ring with the maximal ideal $\mathfrak{n} \widehat{\mathcal{E}}(\mathfrak{n})$.) Take $L$ as in the lemma and put $v={ }^{t}\left(v_{1}, v_{2}\right)=L^{-1} u$. Since $\left(H 1_{2}-Q-R E_{+}^{2}\right) u=0$, we have $\left(H 1_{2}-Q-T\right) v=0$ by condition (1). The action of $H$ on $v$ is easier than that on $u$ (for example, $v_{2}$ is an $H$-eigenvector) since $Q+T$ is an upper triangular matrix.

Proof of Lemma 2.3. From condition (1), we have

$$
\left[H 1_{2}, L\right]-[Q, L]=R E_{+}^{2} L-L T .
$$


The calculation of the left hand side is not so difficult. In fact, the term $\left[H 1_{2}, L\right]$ can be calculated using the $H$-weight decomposition, and $[Q, L]$ calculated easily since $Q$ is a diagonal matrix.

Thus, take $L_{k}, T_{k} \in M(2, \mathbb{C})$ such that $L=\sum_{k} L_{k} E_{+}^{2 k}, T=\sum_{k} T_{k} E_{+}^{2 k}$. Moreover, define $a_{k}, b_{k}, c_{k}, d_{k} \in \mathbb{C}$ by

$$
L_{k}=\left(\begin{array}{cc}
a_{k} & b_{k} \\
c_{k} & d_{k}
\end{array}\right) .
$$

Then the left hand side of (2.1) is

$$
\sum_{k=0}^{\infty}\left(\begin{array}{cc}
2 k a_{k} & 2(k-\lambda) b_{k} \\
2(k+\lambda) c_{k} & 2 k d_{k}
\end{array}\right) E_{+}^{2 k}
$$

On the other hand, the right hand side of (2.1) is not so easy. We only expand the right hand side of (2.1) into a power series. Namely, the right hand side of $(2.1)$ is

$$
\sum_{k=0}^{\infty}\left(R L_{k-1}-\sum_{l=0}^{k} L_{l} T_{k-l}\right) E_{+}^{2 k}
$$

where $L_{-1}=0$. By the conditions, we have $T_{0}=0$ and $L_{0}=1_{2}$. Hence the right hand side of (2.1) is

$$
\sum_{k=0}^{\infty}\left(R L_{k-1}-\sum_{l=1}^{k-1} L_{l} T_{k-l}-T_{k}\right) E_{+}^{2 k} .
$$

Therefore $\left(H-Q-R E_{+}^{2}\right) L=L(H-Q-T)$ is equivalent to

$$
\left(\begin{array}{cc}
2 k a_{k} & 2(k-\lambda) b_{k} \\
2(k+\lambda) c_{k} & 2 k d_{k}
\end{array}\right)+T_{k}=R L_{k-1}-\sum_{l=1}^{k-1} L_{l} T_{k-l}
$$

for all $k>0$. (The case of $k=0$ is automatically satisfied.)

We take $L_{k}$ and $T_{k}$ inductively. Assume that we have already chosen $L_{0}, \ldots, L_{k-1}$ and $T_{0}, \ldots, T_{k-1}$. Then the right hand side of $(2.2)$ is determined. If $\lambda \neq k$, then we can take $a_{k}, b_{k}, c_{k}, d_{k}$ such that $T_{k}=0$. If $\lambda=k$, then we can take $a_{k}, b_{k}, c_{k}, d_{k}$ such that

$$
T_{k}=\left(\begin{array}{ll}
0 & t \\
0 & 0
\end{array}\right)
$$

for some $t \in \mathbb{C}$. (Recall that we assume $\operatorname{Re} \lambda \geq 0$ and $\lambda \neq 0$.) Hence the lemma is proved. 
Take $L$ as in the lemma and put $v={ }^{t}\left(v_{1}, v_{2}\right)=L^{-1} u$. As already mentioned, $\left(H 1_{2}-Q-T\right) v=0$. In particular, we have $H v_{2}=(-\lambda+1 / 2) v_{2}$. Moreover, since $(H-(\lambda+1 / 2)) v_{1}=t E_{+}^{2 \lambda} v_{2}$ for some $t \in \mathbb{C}$, we have $(H-(\lambda+$ $1 / 2))^{2} v_{1}=t(H-(\lambda+1 / 2)) E_{+}^{2 \lambda} v_{2}=t E_{+}^{2 \lambda}(H-(-\lambda+1 / 2)) v_{2}=0$. Namely, $v_{1}$ has a generalized $H$-eigenvalue $\lambda+1 / 2$.

We prove that $v_{1}$ and $v_{2}$ generate $\widehat{J}(I(\lambda))$ as an $\widehat{\mathcal{E}}(\mathfrak{n})$-module. By Nakayama's lemma, it is sufficient to prove that $v_{1}+\mathfrak{n} \widehat{J}(I(\lambda))$ and $v_{2}+\mathfrak{n} \widehat{J}(I(\lambda))$ generate $\widehat{J}(I(\lambda)) / \mathfrak{n} \widehat{J}(I(\lambda))$. However, since $L \in 1_{2}+M(2, \mathfrak{n} \widehat{\mathcal{E}}(\mathfrak{n}))$, we have $v_{i} \equiv u_{i}(\bmod \mathfrak{n} \widehat{J}(I(\lambda)))$. Since the set of images of $u_{1}, u_{2}$ in $I(\lambda) / \mathfrak{n} I(\lambda)$ is a basis of $I(\lambda) / \mathfrak{n} I(\lambda) \simeq \widehat{J}(I(\lambda)) / \mathfrak{n} \widehat{J}(I(\lambda)), v_{1}+\mathfrak{n} \widehat{J}(I(\lambda))$ and $v_{2}+\widehat{J}(I(\lambda))$ generate $\widehat{J}(I(\lambda)) / \mathfrak{n} \widehat{J}(I(\lambda))$.

Now we prove the following lemma.

\section{Lemma 2.4 .}

(1) $J(I(\lambda))=U(\mathfrak{g}) v_{1}+U(\mathfrak{g}) v_{2}$.

(2) $E_{-} v_{2}=0$.

(3) $E_{-} v_{1} \in U(\mathfrak{g}) v_{2}$

From Lemma 2.4, $v_{2}$ is a lowest weight vector in $J(I(\lambda))$ and $v_{1}$ is a lowest weight vector in $J(I(\lambda)) / U(\mathfrak{g}) v_{2}$.

To prove these formulae, we use the projection to a generalized $H$ eigenspace. The justification will be done in $\S 3$ (Corollary 3.9). Recall that the generalized $H$-eigenvalue of $v_{1}$ (resp. $\left.v_{2}\right)$ is $\lambda+1 / 2($ resp. $-\lambda+1 / 2)$.

Proof of Lemma 2.4. First we prove (1). Let $v \in J(I(\lambda))$ be a generalized eigenvector of $H$ with a generalized eigenvalue $\mu$. Since $v_{1}, v_{2}$ generate $\widehat{J}(I(\lambda))$ as an $\widehat{\mathcal{E}}(\mathfrak{n})$-module, there exist $B^{(1)}=\sum_{k} B_{k}^{(1)} E_{+}^{k} \in \widehat{\mathcal{E}}(\mathfrak{n})$ and $B^{(2)}=\sum_{k} B_{k}^{(2)} E_{+}^{k} \in \widehat{\mathcal{E}}(\mathfrak{n})$ such that $v=B^{(1)} v_{1}+B^{(2)} v_{2}$. Applying the projection to the generalized $H$-eigenspace with an eigenvalue $\mu$, we have $v=B_{\mu-\lambda-1 / 2}^{(1)} E_{+}^{\mu-\lambda-1 / 2} v_{1}+B_{\mu+\lambda-1 / 2}^{(2)} E_{+}^{\mu+\lambda-1 / 2} v_{2}$ (Here $E_{+}^{t}=0$ if $t \notin \mathbb{Z}_{\geq 0}$ ). This implies $v \in U(\mathfrak{g}) v_{1}+U(\mathfrak{g}) v_{2}$.

We will calculate $E_{-} v_{1}$ and $E_{-} v_{2}$. Roughly speaking, these equations are induced from $\left(E_{+}-E_{-}\right) u_{\lambda}=0$ and the projection to a generalized $H$ eigenspace. Take $A^{(1)}, A^{(2)} \in \widehat{\mathcal{E}}(\mathfrak{n})$ such that $u_{\lambda}=A^{(1)} v_{1}+A^{(2)} v_{2}$. Take $A_{k}^{(i)} \in \mathbb{C}$ such that $A^{(i)}=\sum A_{k}^{(i)} E_{+}^{k}$. From $\left(E_{-}-E_{+}\right) u_{\lambda}=0$, we have 


$$
\begin{aligned}
0 & =A_{0}^{(1)} E_{-} v_{1}+\sum_{k=1}^{\infty} A_{k}^{(1)} E_{-} E_{+}^{k} v_{1}-\sum_{k=0}^{\infty} A_{k}^{(1)} E_{+}^{k+1} v_{1} \\
& +A_{0}^{(2)} E_{-} v_{2}+\sum_{k=1}^{\infty} A_{k}^{(2)} E_{-} E_{+}^{k} v_{2}-\sum_{k=0}^{\infty} A_{k}^{(2)} E_{+}^{k+1} v_{2} .
\end{aligned}
$$

Recall that $v_{1}$ (resp. $v_{2}$ ) has a generalized $H$-eigenvalue $\lambda+1 / 2$ (resp. $-\lambda+1 / 2$ ). Hence the generalized $H$-eigenvalue of each term is the following:

$$
\begin{gathered}
\lambda+1 / 2-1, \quad \lambda+1 / 2-1+k, \quad \lambda+1 / 2+(k+1), \\
-\lambda+1 / 2-1,-\lambda+1 / 2-1+k,-\lambda+1 / 2+(k+1) .
\end{gathered}
$$

Using the projection to the generalized $H$-eigenspace with an eigenvalue $-\lambda+$ $1 / 2-1$, we get $A_{0}^{(2)} E_{-} v_{2}=0$ since $\operatorname{Re} \lambda \geq 0$.

Next, we project both sides to the generalized $H$-eigenspace with an eigenvalue $\lambda+1 / 2-1$. If $\lambda \notin \mathbb{Z} / 2$, then $A_{0}^{(1)} E_{-} v_{1}=0$. If $\lambda \in \mathbb{Z} / 2$, we get

$$
0=A_{0}^{(1)} E_{-} v_{1}+A_{2 \lambda}^{(2)} E_{-} E_{+}^{2 \lambda} v_{2}-A_{2 \lambda-2}^{(2)} E_{+}^{2 \lambda-1} v_{2} .
$$

Hence we have $A_{0}^{(1)} E_{-} v_{1} \in U(\mathfrak{g}) v_{2}$.

We must prove that $A_{0}^{(1)}, A_{0}^{(2)} \neq 0$. Since $u_{\lambda}=A^{(1)} v_{1}+A^{(2)} v_{2}$, we have $u_{\lambda} \equiv A_{0}^{(1)} v_{1}+A_{0}^{(2)} v_{2}(\bmod \mathfrak{n} \widehat{J}(I(\lambda)))$. Moreover, since $L \in 1+M(2, \mathfrak{n} \widehat{\mathcal{E}}(\mathfrak{n}))$, we have $u_{i} \equiv v_{i}(\bmod \mathfrak{n} \widehat{J}(I(\lambda)))$. Hence we have $u_{\lambda} \equiv A_{0}^{(1)} u_{1}+A_{0}^{(2)} u_{2}$ $(\bmod \mathfrak{n} \widehat{J}(I(\lambda)))$. By the definition of $u_{1}, u_{2}, u_{\lambda}=(1 / 2 \lambda) u_{1}+(-1 / 2 \lambda) u_{2}$. Since $\left\{u_{1}+\mathfrak{n} I(\lambda), u_{2}+\mathfrak{n} I(\lambda)\right\}$ is a basis of $I(\lambda) / \mathfrak{n} I(\lambda) \simeq \widehat{J}(I(\lambda)) / \mathfrak{n} \widehat{J}(I(\lambda))$, we have $A_{0}^{(1)}=1 / 2 \lambda$ and $A_{0}^{(2)}=-1 / 2 \lambda$. These are nonzero.

Recall the definition of Verma module. For $\mu \in \mathbb{C}$, let $\mathbb{C}_{\mu}$ be the 1dimensional representation of $\mathbb{C} H+\mathbb{C} E_{-}$such that $H v=\mu v$ and $E_{-} v=0$ for $v \in \mathbb{C}_{\mu}$. Put $M(\mu)=U(\mathfrak{g}) \otimes_{U\left(\mathbb{C} H+\mathbb{C} E_{-}\right)} \mathbb{C}_{\mu+1 / 2}$ and $V=U(\mathfrak{g}) v_{2}$. Since $v_{2}$ is a lowest weight vector of $J(I(\lambda))$ and $v_{1}$ is a lowest weight vector of $J(I(\lambda)) / V$, we have surjective maps $M(-\lambda) \rightarrow V$ and $M(\lambda) \rightarrow J(I(\lambda)) / V$.

To prove that these maps are isomorphism, we use the Osborne conjecture, which is now a theorem [HS83a]. Using the Osborne conjecture, we can calculate the character of $J(I(\lambda))$ from the character of $I(\lambda)$ (see Section 5). As a consequence, we have $\Theta(J(I(\lambda)))=\Theta(M(\lambda))+\Theta(M(-\lambda))$ where $\Theta$ denotes the character. Hence we have $V \simeq M(-\lambda)$ and $J(I(\lambda)) / V=M(\lambda)$.

In the rest of this paper, we generalize these arguments to the general case. 


\section{$\S 3 . \quad$ Jacquet Modules and Fundamental Properties}

We now treat the general case. Set $\widehat{\mathcal{E}}(\mathfrak{n})=\varliminf_{\leftarrow} U(\mathfrak{n}) / \mathfrak{n}^{k} U(\mathfrak{n})$. For a projective system $\left\{M_{k}, \varphi_{k}: M_{k} \rightarrow M_{k-1}\right\}_{k \in \mathbb{Z}_{\geq 0}}$, we construct the projective limit as $\varliminf_{k} M_{k}=\left\{\left(m_{k}\right)_{k \in \mathbb{Z}_{\geq 0}} \mid m_{k} \in M_{k}, \varphi_{k}\left(m_{k}\right)=m_{k-1}\right\}$.

\section{Proposition 3.1.}

(1) The $\mathbb{C}$-algebra $\widehat{\mathcal{E}}(\mathfrak{n})$ is right and left Noetherian.

(2) The $\mathbb{C}$-algebra $\widehat{\mathcal{E}}(\mathfrak{n})$ is flat over $U(\mathfrak{n})$.

(3) If $\mathbb{X}$ is a finitely generated $U(\mathfrak{n})$-module then $\varliminf_{k} \mathbb{X} / \mathfrak{n}^{k} \mathbb{X}=\widehat{\mathcal{E}}(\mathfrak{n}) \otimes_{U(\mathfrak{n})} \mathbb{X}$.

(4) Let $S=\left(S_{k}\right)_{k \in \mathbb{Z}_{\geq 0}}$ be an element of $M(r, \mathfrak{n} \widehat{\mathcal{E}}(\mathfrak{n}))$ and $\left(a_{n}\right) \in \mathbb{C}^{\mathbb{Z} \geq 0}$. Define $\sum_{n=0}^{\infty} a_{n} S^{n}=\left(\sum_{n=0}^{k} a_{n} S_{k}^{n}\right)_{k}$. Then $\sum_{n=0}^{\infty} a_{n} S^{n} \in M(r, \widehat{\mathcal{E}}(\mathfrak{n}))$.

Proof. Since Stafford and Wallach [SW82, Theorem 2.1] show that $\mathfrak{n} U(\mathfrak{n})$ $\subset U(\mathfrak{n})$ satisfies the Artin-Rees property, the usual argument of the proof for commutative rings can be applied to prove (1), (2) and (3). (4) is obvious.

Corollary 3.2. Let $S$ be an element of $M(r, \widehat{\mathcal{E}}(\mathfrak{n}))$ such that $S-1_{r} \in$ $M(r, \mathfrak{n} \widehat{\mathcal{E}}(\mathfrak{n}))$. Then $S$ is invertible.

Proof. Set $T=1_{r}-S$. By Proposition 3.1, $R=\sum_{n=0}^{\infty} T^{n} \in M(r, \widehat{\mathcal{E}}(\mathfrak{n}))$. Then $S R=R S=1_{r}$.

We can prove the following proposition using the methods of Goodman and Wallach [GW80, Lemma 2.2]. For the sake of completeness we give a proof.

Proposition 3.3. Let $\mathbb{X}$ be $a U(\mathfrak{a} \oplus \mathfrak{n})$-module which is finitely generated as a $U(\mathfrak{n})$-module. Assume that every element of $\mathbb{X}$ is $\mathfrak{a}$-finite. For $\mu \in \mathfrak{a}^{*}$ set

$$
\begin{gathered}
\mathbb{X}_{\mu}=\{x \in \mathbb{X} \mid \text { For all } H \in \mathfrak{a} \text { there exists a positive integer } N \text { such that } \\
\left.\qquad(H-\mu(H))^{N} x=0\right\} .
\end{gathered}
$$

Then

$$
\widehat{J}(\mathbb{X}) \simeq \prod_{\mu \in \mathfrak{a}^{*}} \mathbb{X}_{\mu}
$$

Proof. Since the action of $\mathfrak{n}$ increases an $\mathfrak{a}$-weight and $\mathbb{X}$ is a finitely generated $U(\mathfrak{n})$-module, there exists a positive integer $k_{\mu}$ for all $\mu$ such that

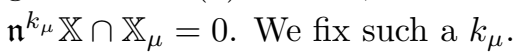


For $k \in \mathbb{Z}_{>0}$ put $S_{k}=\left\{\mu \in \mathfrak{a}^{*} \mid \mathbb{X}_{\mu} \neq 0, \mathbb{X}_{\mu} \not \subset \mathfrak{n}^{k} \mathbb{X}\right\}$. Since $\mathbb{X}$ is finitely generated, $\operatorname{dim} \mathbb{X} / \mathfrak{n}^{k} \mathbb{X}<\infty$. Therefore $S_{k}$ is a finite set. Define a map $\varphi: \prod_{\mu \in \mathfrak{a}^{*}} \mathbb{X}_{\mu} \rightarrow \widehat{J}(\mathbb{X})$ by

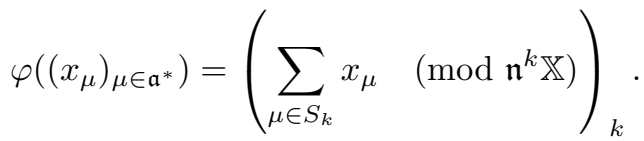

First we show that $\varphi$ is injective. Assume $\varphi\left(\left(x_{\mu}\right)_{\mu \in \mathfrak{a}^{*}}\right)=0$. We have $\sum_{\mu \in S_{k}} x_{\mu} \in \mathfrak{n}^{k} \mathbb{X}$ for all $k \in \mathbb{Z}_{>0}$. Since $\mathfrak{n}^{k} \mathbb{X}$ is $\mathfrak{a}$-stable and $S_{k}$ is a finite set, $x_{\mu} \in \mathfrak{n}^{k} \mathbb{X}$ for all $\mu \in \mathfrak{a}^{*}$ and $k \in \mathbb{Z}_{>0}$. In particular, we have $x_{\mu} \in \mathbb{X}_{\mu} \cap \mathfrak{n}^{k_{\mu}} \mathbb{X}=0$.

We have to show that $\varphi$ is surjective. Let $x=\left(x_{k}\left(\bmod \mathfrak{n}^{k} \mathbb{X}\right)\right)_{k}$ be an element of $\widehat{J}(\mathbb{X})$. Since every element of $\mathbb{X}$ is $\mathfrak{a}$-finite, we have $\mathbb{X}=\bigoplus_{\mu \in \mathfrak{a}^{*}} \mathbb{X}_{\mu}$. Let $p_{\mu}: \mathbb{X} \rightarrow \mathbb{X}_{\mu}$ be the projection. Notice that if $i, i^{\prime} \geq k_{\mu}$ then $p_{\mu}\left(x_{i}\right)=$ $p_{\mu}\left(x_{i^{\prime}}\right)$. Hence we have $\varphi\left(\left(p_{\mu}\left(x_{k_{\mu}}\right)\right)_{\mu \in \mathfrak{a}^{*}}\right)=x$.

We define an $(\mathfrak{a} \oplus \mathfrak{n})$-representation structure of $U(\mathfrak{n})$ by $(H+X)(u)=$ $H u-u H+X u$ for $H \in \mathfrak{a}, X \in \mathfrak{n}, u \in U(\mathfrak{n})$. Then $U(\mathfrak{n})$ is a $U(\mathfrak{a} \oplus \mathfrak{n})$-module. By Proposition 3.3, $\widehat{\mathcal{E}}(\mathfrak{n})=\prod_{\mu \in \mathfrak{a}^{*}} U(\mathfrak{n})_{\mu}$. The following results are corollaries of Proposition 3.3.

Corollary 3.4. The linear map

$$
\begin{aligned}
\mathbb{C}\left[\left[X_{1}, X_{2}, \ldots, X_{m}\right]\right] & \widehat{\mathcal{E}}(\mathfrak{n}) \\
\sum_{\mathbf{n} \in \mathbb{Z}_{\geq 0}^{m}} a_{\mathbf{n}} X^{\mathbf{n}} & \longmapsto\left(\sum_{|\mathbf{n}| \leq k} a_{\mathbf{n}} E^{\mathbf{n}} \quad\left(\bmod \mathfrak{n}^{k} U(\mathfrak{n})\right)\right)_{k}
\end{aligned}
$$

is bijective, where $X^{\mathbf{n}}=X_{1}^{\mathbf{n}_{1}} X_{2}^{\mathbf{n}_{2}} \cdots X_{m}^{\mathbf{n}_{m}}$ for $\mathbf{n}=\left(\mathbf{n}_{1}, \mathbf{n}_{2}, \ldots, \mathbf{n}_{m}\right) \in \mathbb{Z}_{\geq 0}^{m}$.

Proof. By the Poincaré-Birkhoff-Witt theorem, $\left\{E^{\mathbf{n}} \mid \sum_{i} \mathbf{n}_{i} \beta_{i}=\mu\right\}$ is a basis of $U(\mathfrak{n})_{\mu}$. This implies the corollary since $\widehat{\mathcal{E}}(\mathfrak{n})=\prod_{\mu \in \mathfrak{a}^{*}} U(\mathfrak{n})_{\mu}$.

We denote the image of $\sum_{\mathbf{n} \in \mathbb{Z}_{>0}^{m}} a_{\mathbf{n}} X^{\mathbf{n}}$ under the map in Corollary 3.4 by $\sum_{\mathbf{n} \in \mathbb{Z}_{\geq 0}^{m}} a_{\mathbf{n}} E^{\mathbf{n}}$.

Corollary 3.5. Let $\mathbb{X}$ be a $U(\mathfrak{g})$-module which is finitely generated as a $U(\mathfrak{n})$-module. Assume that all elements are $\mathfrak{a}$-finite. Then $J(\mathbb{X})=\mathbb{X}$.

Proof. This follows from the following equation.

$$
J(\mathbb{X})=\widehat{J}(\mathbb{X})_{\mathfrak{a} \text {-finite }}=\left(\prod_{\mu \in \mathfrak{a}^{*}} \mathbb{X}_{\mu}\right)_{\mathfrak{a} \text {-finite }}=\bigoplus_{\mu \in \mathfrak{a}^{*}} \mathbb{X}_{\mu}=\mathbb{X}
$$


Put $\widehat{\mathcal{E}}(\mathfrak{g}, \mathfrak{n})=\widehat{\mathcal{E}}(\mathfrak{n}) \otimes_{U(\mathfrak{n})} U(\mathfrak{g})$. We can define a $\mathbb{C}$-algebra structure of $\widehat{\mathcal{E}}(\mathfrak{g}, \mathfrak{n})$ by

$$
\begin{aligned}
(f \otimes 1)(1 \otimes u) & =f \otimes u, \\
(1 \otimes u)\left(1 \otimes u^{\prime}\right) & =1 \otimes\left(u u^{\prime}\right), \\
(f \otimes 1)\left(f^{\prime} \otimes 1\right) & =\left(f f^{\prime}\right) \otimes 1, \\
(1 \otimes X)(f \otimes 1) & =\sum_{\mathbf{n} \in \mathbb{Z}_{\geq 0}^{r}} \frac{1}{\mathbf{n} !} \frac{\partial^{|\mathbf{n}|}}{\partial E^{\mathbf{n}}} f \otimes^{t}\left((\operatorname{ad}(E))^{\mathbf{n}}\right)(X),
\end{aligned}
$$

where $u, u^{\prime} \in U(\mathfrak{g}), X \in \mathfrak{g}, f, f^{\prime} \in \widehat{\mathcal{E}}(\mathfrak{n}),{ }^{t}\left((\operatorname{ad}(E))^{\mathbf{n}}\right)=\left(-\operatorname{ad}\left(E_{m}\right)\right)^{\mathbf{n}_{m}} \ldots$ $\left(-\operatorname{ad}\left(E_{1}\right)\right)^{\mathbf{n}_{1}}$ and

$$
\frac{\partial^{|\mathbf{n}|}}{\partial E^{\mathbf{n}}}\left(\sum_{\mathbf{m} \in \mathbb{Z}_{\geq 0}^{m}} a_{\mathbf{m}} E^{\mathbf{m}}\right)=\sum_{\mathbf{m} \in \mathbb{Z}_{\geq 0}^{m}} a_{\mathbf{m}} \frac{\mathbf{m} !}{(\mathbf{m}-\mathbf{n}) !} E^{\mathbf{m}-\mathbf{n}} .
$$

It is not difficult to see that this definition is independent of a choice of a basis $\left\{E_{i}\right\}$ and its order. However, we do not use it. So we omit the proof.

Notice that $\widehat{\mathcal{E}}(\mathfrak{g}, \mathfrak{n}) \otimes_{U(\mathfrak{g})} \mathbb{X} \simeq \widehat{\mathcal{E}}(\mathfrak{n}) \otimes_{U(\mathfrak{n})} \mathbb{X}$ as an $\widehat{\mathcal{E}}(\mathfrak{n})$-module for a $U(\mathfrak{g})$ module $\mathbb{X}$. By Proposition 3.1, $\widehat{\mathcal{E}}(\mathfrak{g}, \mathfrak{n})$ is flat over $U(\mathfrak{g})$. Notice that if $\mathfrak{b}$ is a subalgebra of $\mathfrak{g}$ which contains $\mathfrak{n}$ then $\widehat{\mathcal{E}}(\mathfrak{n}) \otimes_{U(\mathfrak{n})} U(\mathfrak{b})$ is a subalgebra of $\widehat{\mathcal{E}}(\mathfrak{g}, \mathfrak{n})$. Put $\widehat{\mathcal{E}}(\mathfrak{b}, \mathfrak{n})=\widehat{\mathcal{E}}(\mathfrak{n}) \otimes_{U(\mathfrak{n})} U(\mathfrak{b})$.

Let $\mathbb{X}$ be a $U(\mathfrak{a} \oplus \mathfrak{n})$-module such that $\mathbb{X}=\bigoplus_{\mu \in \mathfrak{a}^{*}} \mathbb{X}_{\mu}$. Set

$$
\begin{array}{r}
V=\left\{\left(x_{\mu}\right)_{\mu} \in \prod_{\mu \in \mathfrak{a}^{*}} \mathbb{X}_{\mu} \mid\right. \text { there exists an element } \\
\left.\nu \in \mathfrak{a}_{0}^{*} \text { such that } x_{\mu}=0 \text { for } \operatorname{Re} \mu<\nu\right\} .
\end{array}
$$

Then we can define an $\mathfrak{a}$-module homomorphism

$$
\varphi: \widehat{\mathcal{E}}(\mathfrak{a} \oplus \mathfrak{n}, \mathfrak{n}) \otimes_{U(\mathfrak{a} \oplus \mathfrak{n})} \mathbb{X} \simeq\left(\prod_{\mu \in \mathfrak{a}^{*}} U(\mathfrak{n})_{\mu}\right) \otimes_{U(\mathfrak{n})}\left(\bigoplus_{\mu^{\prime} \in \mathfrak{a}^{*}} \mathbb{X}_{\mu^{\prime}}\right) \rightarrow V
$$

by $\varphi\left(\left(f_{\mu}\right)_{\mu \in \mathfrak{a}^{*}} \otimes\left(x_{\mu^{\prime}}\right)_{\mu^{\prime} \in \mathfrak{a}^{*}}\right)=\left(\sum_{\mu+\mu^{\prime}=\lambda} f_{\mu} x_{\mu^{\prime}}\right)_{\lambda \in \mathfrak{a}^{*}}$. Notice that the composition of the maps $\mathbb{X} \rightarrow \widehat{\mathcal{E}}(\mathfrak{a} \oplus \mathfrak{n}, \mathfrak{n}) \otimes_{U(\mathfrak{a} \oplus \mathfrak{n})} \mathbb{X} \rightarrow V$ is equal to the inclusion map $\mathbb{X} \hookrightarrow V$.

We consider the case $\mathbb{X}=U(\mathfrak{g})$. Define an $(\mathfrak{a} \oplus \mathfrak{n})$-module structure of $U(\mathfrak{g})$ by $(H+X)(u)=H u-u H+X u$ for $H \in \mathfrak{a}, X \in \mathfrak{n}, u \in U(\mathfrak{g})$. We have a map 


$$
\begin{array}{r}
\varphi: \widehat{\mathcal{E}}(\mathfrak{g}, \mathfrak{n}) \rightarrow \\
\left\{\left(P_{\mu}\right)_{\mu \in \mathfrak{a}^{*}} \in \prod_{\mu \in \mathfrak{a}^{*}} U(\mathfrak{g})_{\mu} \mid\right. \text { there exists an element } \\
\left.\nu \in \mathfrak{a}_{0}^{*} \text { such that } P_{\mu}=0 \text { for } \operatorname{Re} \mu<\nu\right\} .
\end{array}
$$

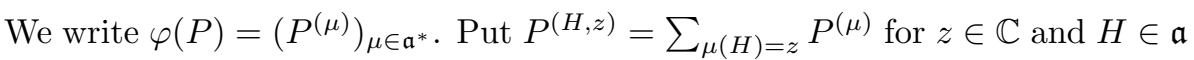
such that $\operatorname{Re} \alpha(H)>0$ for all $\alpha \in \Sigma^{+}$. By the condition on $H$, the right hand side is a finite sum.

Proposition 3.6 and 3.7 follow at once from the definition.

Proposition 3.6. Let $\mathbb{X}$ be a $U(\mathfrak{a} \oplus \mathfrak{n})$-module such that $\mathbb{X}$ is finitely generated as a $U(\mathfrak{n})$-module and each element is $\mathfrak{a}$-finite. Let $\varphi: \widehat{\mathcal{E}}(\mathfrak{a} \oplus \mathfrak{n}, \mathfrak{n}) \otimes_{U(\mathfrak{a} \oplus \mathfrak{n})}$ $\mathbb{X} \rightarrow \prod_{\mu \in \mathfrak{a}^{*}} \mathbb{X}_{\mu}$ be the $\mathfrak{a}$-module homomorphism defined as above. Then $\varphi$ coincides with the map given in Proposition 3.3. In particular $\varphi$ is isomorphic.

\section{Proposition 3.7.}

(1) We have $(P Q)^{(\lambda)}=\sum_{\mu+\mu^{\prime}=\lambda} P^{(\mu)} Q^{\left(\mu^{\prime}\right)}$ for $P, Q \in \widehat{\mathcal{E}}(\mathfrak{g}, \mathfrak{n})$ and $\lambda \in \mathfrak{a}^{*}$. (Since $P^{(\mu)}=0$ (resp. $Q^{\left(\mu^{\prime}\right)}=0$ ) if $\operatorname{Re} \mu$ (resp. Re $\left.\mu^{\prime}\right)$ is sufficiently small, the right hand side is a finite sum.)

(2) We have

$$
\left(\sum_{\mathbf{n} \in \mathbb{Z}_{\geq 0}^{m}} a_{\mathbf{n}} E^{\mathbf{n}}\right)^{(\lambda)}=\sum_{\sum_{i} \mathbf{n}_{i} \beta_{i}=\lambda} a_{\mathbf{n}} E^{\mathbf{n}}
$$

for $\lambda \in \mathfrak{a}^{*}$.

Proposition 3.8. Let $\mathbb{X}$ be a $U(\mathfrak{g})$-module which is finitely generated as a $U(\mathfrak{n})$-module. We take generators $v_{1}, v_{2}, \ldots, v_{n}$ of an $\widehat{\mathcal{E}}(\mathfrak{g}, \mathfrak{n})$-module $\widehat{\mathcal{E}}(\mathfrak{g}, \mathfrak{n}) \otimes_{U(\mathfrak{g})} \mathbb{X}$ and set $V=\sum_{i} U(\mathfrak{g}) v_{i} \subset \widehat{\mathcal{E}}(\mathfrak{g}, \mathfrak{n}) \otimes_{U(\mathfrak{g})} \mathbb{X}$. Define a surjective $\operatorname{map} \psi: \widehat{\mathcal{E}}(\mathfrak{g}, \mathfrak{n}) \otimes_{U(\mathfrak{g})} V \rightarrow \widehat{\mathcal{E}}(\mathfrak{g}, \mathfrak{n}) \otimes_{U(\mathfrak{g})} \mathbb{X}$ by $\psi(f \otimes v)=$ fv. Assume that there exist weights $\lambda_{i} \in \mathfrak{a}^{*}$ and a positive integer $N$ such that $\left(H-\lambda_{i}(H)\right)^{N} v_{i}=0$ for all $H \in \mathfrak{a}$ and $1 \leq i \leq n$. Let $\varphi: \widehat{\mathcal{E}}(\mathfrak{g}, \mathfrak{n}) \otimes_{U(\mathfrak{g})} V \rightarrow \prod_{\mu \in \mathfrak{a}^{*}} V_{\mu}$ be the map defined as above. Then there exists a unique map $\widehat{\mathcal{E}}(\mathfrak{g}, \mathfrak{n}) \otimes_{U(\mathfrak{g})} \mathbb{X} \rightarrow \prod_{\mu \in \mathfrak{a}^{*}} V_{\mu}$ such that the diagram

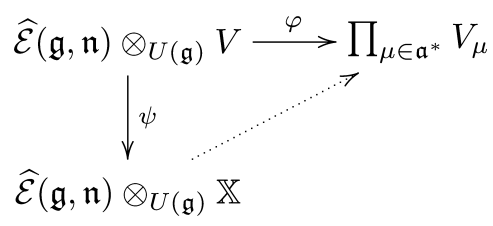


is commutative.

Proof. $\quad$ Set $\widehat{\mathbb{X}}=\widehat{\mathcal{E}}(\mathfrak{g}, \mathfrak{n}) \otimes_{U(\mathfrak{g})} \mathbb{X}$ and $\widehat{V}=\widehat{\mathcal{E}}(\mathfrak{g}, \mathfrak{n}) \otimes_{U(\mathfrak{g})} V$. Take $f^{(i)} \in$ $\widehat{\mathcal{E}}(\mathfrak{g}, \mathfrak{n})$ and $v^{(i)} \in V$ such that $\psi\left(\sum_{i} f^{(i)} \otimes v^{(i)}\right)=0$. We have to show $\varphi\left(\sum_{i} f^{(i)} \otimes v^{(i)}\right)=0$. Since $\widehat{V}=\widehat{\mathcal{E}}(\mathfrak{n}) \otimes_{U(\mathfrak{n})} V$, we may assume $f^{(i)} \in \widehat{\mathcal{E}}(\mathfrak{n})$. We can write $f^{(i)}=\left(f_{\mu}^{(i)}\right)_{\mu \in \mathfrak{a}^{*}}$ by the isomorphism $\widehat{\mathcal{E}}(\mathfrak{n}) \simeq \prod_{\mu \in \mathfrak{a}^{*}} U(\mathfrak{n})_{\mu}$. Since $V=\bigoplus_{\mu^{\prime} \in \mathfrak{a}^{*}} V_{\mu^{\prime}}$, we can write $v^{(i)}=\sum_{\mu^{\prime} \in \mathfrak{a}^{*}} v_{\mu^{\prime}}^{(i)}, v_{\mu^{\prime}}^{(i)} \in V_{\mu^{\prime}}$. We have to show $\sum_{i} \sum_{\mu+\mu^{\prime}=\lambda} f_{\mu}^{(i)} v_{\mu^{\prime}}^{(i)}=0$ for all $\lambda \in \mathfrak{a}^{*}$. Since $\mathbb{X}$ is a finitely generated $U(\mathfrak{n})$-module we have $\widehat{\mathbb{X}}=\lim _{k} \mathbb{X} / \mathfrak{n}^{k} \mathbb{X}=\varliminf_{k} \widehat{\mathbb{X}} / \mathfrak{n}^{k} \widehat{\mathbb{X}}$. It is sufficient to prove $\sum_{i} \sum_{\mu+\mu^{\prime}=\lambda} f_{\mu}^{(i)} v_{\mu^{\prime}}^{(i)} \in \mathfrak{n}^{k} \widehat{\mathbb{X}}$ for all $k \in \mathbb{Z}_{>0}$

Fix $\lambda \in \mathfrak{a}^{*}$ and $k \in \mathbb{Z}_{>0}$. We can choose an element $\nu \in \mathfrak{a}_{0}^{*}$ such that $\bigoplus_{\operatorname{Re} \mu \geq \nu} U(\mathfrak{n})_{\mu} \subset \mathfrak{n}^{k} U(\mathfrak{n})$. Then $0=\psi\left(\sum_{i} f^{(i)} \otimes v^{(i)}\right) \equiv \sum_{i} \sum_{\operatorname{Re} \mu<\nu} f_{\mu}^{(i)} v_{\mu^{\prime}}^{(i)}$ $\left(\bmod \mathfrak{n}^{k} \widehat{\mathbb{X}}\right)$. Notice that the following two sets are finite.

$\left\{\mu \mid \operatorname{Re}(\mu)<\nu\right.$ and there exists an integer $i$ such that $\left.f_{\mu}^{(i)} \neq 0\right\}$, $\left\{\mu^{\prime} \mid\right.$ there exists an integer $i$ such that $\left.v_{\mu^{\prime}}^{(i)} \neq 0\right\}$.

This implies $\sum_{i} \sum_{\mu+\mu^{\prime}=\lambda} f_{\mu}^{(i)} v_{\mu^{\prime}}^{(i)} \in \mathfrak{n}^{k} \widehat{\mathbb{X}}$.

The following result is a corollary of Proposition 3.8.

Corollary 3.9. In the setting of Proposition 3.8, we have the following. Let $P_{i}(1 \leq i \leq n)$ be elements of $\widehat{\mathcal{E}}(\mathfrak{g}, \mathfrak{n})$ such that $\sum_{i=1}^{n} P_{i} v_{i}=0$. Then $\sum_{i} P_{i}^{\left(\lambda-\lambda_{i}\right)} v_{i}=0$ for all $\lambda \in \mathfrak{a}^{*}$.

\section{$\S 4$. Construction of Special Elements}

Let $\Lambda$ be a subset of $\mathcal{P}$. Put $\Lambda^{+}=\Lambda \cap \mathcal{P}^{+}$and $\Lambda^{++}=\Lambda \cap \mathcal{P}^{++}$. We define vector spaces $U(\mathfrak{g})_{\Lambda}, U(\mathfrak{n})_{\Lambda}, \widehat{\mathcal{E}}(\mathfrak{n})_{\Lambda}$ and $\widehat{\mathcal{E}}(\mathfrak{g}, \mathfrak{n})_{\Lambda}$ by

$$
\begin{aligned}
U(\mathfrak{g})_{\Lambda} & =\left\{P \in U(\mathfrak{g}) \mid P^{(\mu)}=0 \text { for all } \mu \notin \Lambda\right\}, \\
U(\mathfrak{n})_{\Lambda} & =\left\{P \in U(\mathfrak{n}) \mid P^{(\mu)}=0 \text { for all } \mu \notin \Lambda\right\}, \\
\widehat{\mathcal{E}}(\mathfrak{n})_{\Lambda} & =\left\{P \in \widehat{\mathcal{E}}(\mathfrak{n}) \mid P^{(\mu)}=0 \text { for all } \mu \notin \Lambda\right\}, \\
\widehat{\mathcal{E}}(\mathfrak{g}, \mathfrak{n})_{\Lambda} & =\left\{P \in \widehat{\mathcal{E}}(\mathfrak{g}, \mathfrak{n}) \mid P^{(\mu)}=0 \text { for all } \mu \notin \Lambda\right\} .
\end{aligned}
$$

Put $(\mathfrak{n} U(\mathfrak{n}))_{\Lambda}=\mathfrak{n} U(\mathfrak{n}) \cap U(\mathfrak{n})_{\Lambda}$ and $(\mathfrak{n} \widehat{\mathcal{E}}(\mathfrak{n}))_{\Lambda}=\mathfrak{n} \widehat{\mathcal{E}}(\mathfrak{n}) \cap \widehat{\mathcal{E}}(\mathfrak{n})_{\Lambda}$

We assume that $\Lambda$ is a subgroup of $\mathfrak{a}^{*}$. Then $U(\mathfrak{g})_{\Lambda}, U(\mathfrak{n})_{\Lambda}, \widehat{\mathcal{E}}(\mathfrak{n})_{\Lambda}$ and $\widehat{\mathcal{E}}(\mathfrak{g}, \mathfrak{n})_{\Lambda}$ are $\mathbb{C}$-algebras. Let $\mathbb{X}$ be a $U(\mathfrak{g})_{\Lambda}$-module which is finitely generated 
as a $U(\mathfrak{n})_{\Lambda}$-module. Let $u_{1}, u_{2}, \ldots, u_{N}$ be generators of $\mathbb{X}$ as a $U(\mathfrak{n})_{\Lambda}$-module. Put $u={ }^{t}\left(u_{1}, u_{2}, \ldots, u_{N}\right), \overline{\mathbb{X}}=\mathbb{X} /(\mathfrak{n} U(\mathfrak{n}))_{\Lambda} \mathbb{X}$ and $\bar{u}=u+(\mathfrak{n} U(\mathfrak{n}))_{\Lambda} \mathbb{X}^{N} \in \overline{\mathbb{X}}^{N}$. The module $\overline{\mathbb{X}}$ has an $\mathfrak{a}$-module structure induced from that of $\mathbb{X}$. Since $\mathbb{X}$ is a finitely generated $U(\mathfrak{n})_{\Lambda}$-module, we have $\operatorname{dim} \overline{\mathbb{X}}<\infty$. Let $\lambda_{1}, \lambda_{2}, \ldots, \lambda_{r} \in \mathfrak{a}^{*}$ $\left(\operatorname{Re} \lambda_{1} \geq \operatorname{Re} \lambda_{2} \geq \cdots \geq \operatorname{Re} \lambda_{r}\right)$ be eigenvalues of $\mathfrak{a}$ on $\overline{\mathbb{X}}$ with multiplicities. We can choose a basis $\overline{v_{1}}, \overline{v_{2}}, \ldots, \overline{v_{r}}$ of $\overline{\mathbb{X}}$ and a linear map $\bar{Q}: \mathfrak{a} \rightarrow M(r, \mathbb{C})$ such that

$$
\left\{\begin{array}{l}
H \bar{v}=\bar{Q}(H) \bar{v} \text { for all } H \in \mathfrak{a}, \\
\bar{Q}(H)_{i i}=\lambda_{i}(H) \text { for all } H \in \mathfrak{a}, \\
\text { if } i>j \text { then } \bar{Q}(H)_{i j}=0 \text { for all } H \in \mathfrak{a}, \\
\text { if } \lambda_{i} \neq \lambda_{j} \text { then } \bar{Q}(H)_{i j}=0 \text { for all } H \in \mathfrak{a},
\end{array}\right.
$$

where $\bar{v}={ }^{t}\left(\overline{v_{1}}, \overline{v_{2}}, \ldots, \overline{v_{r}}\right)$. Take $\bar{C} \in M(N, r, \mathbb{C})$ and $\bar{D} \in M(r, N, \mathbb{C})$ such that $\bar{v}=\bar{D} \bar{u}$ and $\bar{u}=\bar{C} \bar{v}$.

Set $\widehat{\mathbb{X}}=\widehat{\mathcal{E}}(\mathfrak{g}, \mathfrak{n})_{\Lambda} \otimes_{U(\mathfrak{g})_{\Lambda}} \mathbb{X}$.

Theorem 4.1. We use the above notation. There exist matrices $C \in$ $M\left(N, r, \widehat{\mathcal{E}}(\mathfrak{n})_{\Lambda}\right)$ and $D \in M\left(r, N, \widehat{\mathcal{E}}(\mathfrak{n})_{\Lambda}\right)$ such that the following conditions hold:

- There exists a linear map $Q: \mathfrak{a} \rightarrow M\left(r, U(\mathfrak{n})_{\Lambda}\right)$ such that

$$
\left\{\begin{array}{l}
H v=Q(H) v \text { for all } H \in \mathfrak{a}, \\
Q(H)-\bar{Q}(H) \in M\left(r,(\mathfrak{n} U(\mathfrak{n}))_{\Lambda}\right) \text { for all } H \in \mathfrak{a}, \\
\text { if } \lambda_{i}-\lambda_{j} \notin \Lambda^{+} \text {then } Q(H)_{i j}=0 \text { for all } H \in \mathfrak{a}, \\
\text { if } \lambda_{i}-\lambda_{j} \in \Lambda^{+} \text {then }\left[H^{\prime}, Q(H)_{i j}\right]=\left(\lambda_{i}-\lambda_{j}\right)\left(H^{\prime}\right) Q(H)_{i j} \text { for all } H, H^{\prime} \in \mathfrak{a}, \\
\text { if } i>j \text { then } Q(H)_{i j}=0 \text { for all } H \in \mathfrak{a} .
\end{array}\right.
$$

where $v=D u$.

- We have $u=C D u$.

- We have $C-\bar{C} \in M\left(N, r,(\mathfrak{n} \widehat{\mathcal{E}}(\mathfrak{n}))_{\Lambda}\right)$ and $D-\bar{D} \in M\left(r, N,(\mathfrak{n} \widehat{\mathcal{E}}(\mathfrak{n}))_{\Lambda}\right)$.

Remark 4.2. Assume that Theorem 4.1 holds.

(1) Fix $H \in \mathfrak{a}$. Then $Q(H)_{i i}-\lambda_{i}(H)=Q(H)_{i i}-\bar{Q}(H)_{i i} \in(\mathfrak{n} U(\mathfrak{n}))_{\Lambda}$. However we have $\left[H^{\prime}, Q(H)_{i i}-\lambda_{i}(H)\right]=\left[H^{\prime}, Q(H)_{i i}\right]=\left(\lambda_{i}-\lambda_{i}\right)\left(H^{\prime}\right) Q(H)_{i i}=$ 0 for all $H^{\prime} \in \mathfrak{a}$. Hence we have $Q(H)_{i i}=\lambda_{i}(H)$.

(2) We can prove $\left(H-\lambda_{i}(H)\right)^{r-i+1} v_{i}=0$ for all $H \in \mathfrak{a}$ by backward induction on $i$. If $i=r$, then the claim follows from $H v=Q(H) v$ and $Q(H)_{i j}=$ 0 for $i>j$. Assume $i<r$. Then from $H v=Q(H) v$ and (1), we have $\left(H-\lambda_{i}(H)\right) v_{i}=\sum_{j>i} Q(H)_{i j} v_{j}$. Since $\left[H, Q(H)_{i j}\right]=\left(\lambda_{i}-\lambda_{j}\right)(H) Q(H)_{i j}$, we have $\left(H-\lambda_{i}(H)\right)^{r-i+1}=\sum_{j>i} Q(H)_{i j}\left(H-\lambda_{j}(H)\right)^{r-i} v_{j}=0$. 
For the proof we need some lemmas. Put $w=\bar{D} u \in \widehat{\mathbb{X}}^{r}$.

Lemma 4.3. For $H \in \mathfrak{a}$ there exists a matrix $R \in M\left(r,(\mathfrak{n} \widehat{\mathcal{E}}(\mathfrak{n}))_{\Lambda}\right)$ such that $H w=(\bar{Q}(H)+R) w$ in $\widehat{\mathbb{X}}^{r}$.

Proof. Since $w\left(\bmod \left((\mathfrak{n} U(\mathfrak{n}))_{\Lambda} \mathbb{X}\right)^{r}\right)=\bar{v}$, we have $H w-\bar{Q}(H) w \in$ $\left((\mathfrak{n} U(\mathfrak{n}))_{\Lambda} \mathbb{X}\right)^{r}$. Hence there exists a matrix $R_{1} \in M\left(N, r,(\mathfrak{n} U(\mathfrak{n}))_{\Lambda}\right)$ such that $H w-\bar{Q}(H) w=R_{1} u$. Similarly we can choose a matrix $S \in M\left(N,(\mathfrak{n} U(\mathfrak{n}))_{\Lambda}\right)$ which satisfies $u=\bar{C} w+S u$. Put $R=R_{1}(1-S)^{-1} \bar{C}$. Then $(H-\bar{Q}(H)-R) w=$ $R_{1} u-R_{1}(1-S)^{-1} \bar{C} w=0$.

Lemma 4.4. Let $\lambda \in \mathbb{C}$ and $Q_{0}, R_{0} \in M(r, \mathbb{C})$. Assume that $Q_{0}$ is an upper triangular matrix. Then there exist matrices $L_{0}, T_{0} \in M(r, \mathbb{C})$ such that

$$
\left\{\begin{array}{l}
\lambda L_{0}-\left[Q_{0}, L_{0}\right]=T_{0}+R_{0}, \\
\text { if }\left(Q_{0}\right)_{i i}-\left(Q_{0}\right)_{j j} \neq \lambda \text { then }\left(T_{0}\right)_{i j}=0 .
\end{array}\right.
$$

Proof. Since $\left(Q_{0}\right)_{i j}=0$ for $i>j$, we have

$$
\begin{aligned}
\left(\lambda L_{0}-\left[Q_{0}, L_{0}\right]\right)_{i j}= & \lambda\left(L_{0}\right)_{i j}-\sum_{k=1}^{r}\left(\left(Q_{0}\right)_{i k}\left(L_{0}\right)_{k j}-\left(L_{0}\right)_{i k}\left(Q_{0}\right)_{k j}\right) \\
= & \lambda\left(L_{0}\right)_{i j}-\sum_{k=i}^{r}\left(Q_{0}\right)_{i k}\left(L_{0}\right)_{k j}+\sum_{k=1}^{j}\left(L_{0}\right)_{i k}\left(Q_{0}\right)_{k j} \\
= & \left(\lambda-\left(\left(Q_{0}\right)_{i i}-\left(Q_{0}\right)_{j j}\right)\right)\left(L_{0}\right)_{i j}-\sum_{k=i+1}^{r}\left(Q_{0}\right)_{i k}\left(L_{0}\right)_{k j} \\
& +\sum_{k=1}^{j-1}\left(L_{0}\right)_{i k}\left(Q_{0}\right)_{k j} .
\end{aligned}
$$

Hence we can choose $\left(L_{0}\right)_{i j}$ and $\left(T_{0}\right)_{i j}$ inductively on $(j-i)$.

Lemma 4.5. Let $H$ be an element of $\mathfrak{a}$ such that $\alpha(H)>0$ for all $\alpha \in \Sigma^{+}$. Let $Q_{0} \in M(r, \mathbb{C}), R \in M\left(r,(\mathfrak{n} \widehat{\mathcal{E}}(\mathfrak{n}))_{\Lambda}\right)$. Assume $\left(Q_{0}\right)_{i j}=0$ for $i>j$. Set $\mathcal{L}^{++}=\left\{\lambda(H) \mid \lambda \in \Lambda^{++}\right\}$. Then there exist matrices $L \in M\left(r, \widehat{\mathcal{E}}(\mathfrak{n})_{\Lambda}\right)$ and $T \in M\left(r,(\mathfrak{n} U(\mathfrak{n}))_{\Lambda}\right)$ such that

$$
\left\{\begin{array}{l}
L \in 1_{r}+M\left(r,(\mathfrak{n} \widehat{\mathcal{E}}(\mathfrak{n}))_{\Lambda}\right), \\
\left(H 1_{r}-Q_{0}-T\right) L=L\left(H 1_{r}-Q_{0}-R\right), \\
\text { if }\left(Q_{0}\right)_{i i}-\left(Q_{0}\right)_{j j} \notin \mathcal{L}^{++} \text {then } T_{i j}=0, \\
\text { if }\left(Q_{0}\right)_{i i}-\left(Q_{0}\right)_{j j} \in \mathcal{L}^{++} \text {then }\left[H, T_{i j}\right]=\left(\left(Q_{0}\right)_{i i}-\left(Q_{0}\right)_{j j}\right) T_{i j} .
\end{array}\right.
$$


Proof. Set $\mathcal{L}=\{\lambda(H) \mid \lambda \in \Lambda\}$ and $\mathcal{L}^{+}=\left\{\lambda(H) \mid \lambda \in \Lambda^{+}\right\}$. Put $f(\mathbf{n})=\sum_{i} \mathbf{n}_{i} \beta_{i}$ for $\mathbf{n}=\left(\mathbf{n}_{i}\right) \in \mathbb{Z}^{m}$. Set $\widetilde{\Lambda}=\left\{\mathbf{n} \in \mathbb{Z}_{\geq 0}^{m} \mid f(\mathbf{n}) \in \Lambda\right\}$. We define an order on $\widetilde{\Lambda}$ by $\mathbf{n}<\mathbf{n}^{\prime}$ if and only if $f(\mathbf{n})<f\left(\mathbf{n}^{\prime}\right)$.

By Corollary 3.4, we can write $R=\sum_{\mathbf{n} \in \tilde{\Lambda}} R_{\mathbf{n}} E^{\mathbf{n}}$ where $R_{\mathbf{n}} \in M(r, \mathbb{C})$. We have $R_{\mathbf{0}}=0$ where $\mathbf{0}=(0, \ldots, 0) \in \widetilde{\Lambda}$ since $R \in M\left(r,(\mathfrak{n} \widehat{\mathcal{E}}(\mathfrak{n}))_{\Lambda}\right)$. We have to show the existence of $L$ and $T$. Write $L=1_{r}+\sum_{\mathbf{n} \in \tilde{\Lambda}} L_{\mathbf{n}} E^{\mathbf{n}}$ and $T=\sum_{\mathbf{n} \in \tilde{\Lambda}} T_{\mathbf{n}} E^{\mathbf{n}}$. By the conditions of $L$ and $T$, we have $T_{\mathbf{0}}=L_{\mathbf{0}}=0$. Then $\left(H 1_{r}-Q_{0}-T\right) L=L\left(H 1_{r}-Q_{0}-R\right)$ is equivalent to

$$
f(\mathbf{n})(H) L_{\mathbf{n}}-\left[Q_{0}, L_{\mathbf{n}}\right]=T_{\mathbf{n}}+S_{\mathbf{n}}-R_{\mathbf{n}},
$$

where $S_{\mathbf{n}}$ is defined by

$$
\sum_{\mathbf{n} \in \widetilde{\Lambda}} S_{\mathbf{n}} E^{\mathbf{n}}=T\left(L-1_{r}\right)-\left(L-1_{r}\right) R .
$$

By Proposition 3.6, the above equation is equivalent to

$$
\sum_{f(\mathbf{n})=\mu} S_{\mathbf{n}} E^{\mathbf{n}}=\sum_{f(\mathbf{k})+f(\mathbf{l})=\mu} T_{\mathbf{k}} L_{\mathbf{l}} E^{\mathbf{k}} E^{\mathbf{l}}-\sum_{f(\mathbf{k})+f(\mathbf{l})=\mu} L_{\mathbf{k}} R_{\mathbf{l}} E^{\mathbf{k}} E^{\mathbf{l}}
$$

for all $\mu \in \mathfrak{a}^{*} . S_{\mathbf{n}}$ can be defined from the data $\left\{T_{\mathbf{k}} \mid \mathbf{k}<\mathbf{n}\right\},\left\{L_{\mathbf{k}} \mid \mathbf{k}<\mathbf{n}\right\}$ and $\left\{R_{\mathbf{k}} \mid \mathbf{k}<\mathbf{n}\right\}$.

Now we prove the existence of $L$ and $T$. We choose $L_{\mathbf{n}}$ and $T_{\mathbf{n}}$ which satisfy

$$
\left\{\begin{array}{l}
L_{\mathbf{0}}=0, \quad T_{\mathbf{0}}=0, \\
f(\mathbf{n})(H) L_{\mathbf{n}}-\left[Q_{0}, L_{\mathbf{n}}\right]=T_{\mathbf{n}}+S_{\mathbf{n}}-R_{\mathbf{n}}, \\
\text { if }\left(Q_{0}\right)_{i i}-\left(Q_{0}\right)_{j j} \neq f(\mathbf{n})(H) \text { then }\left(T_{\mathbf{n}}\right)_{i j}=0 .
\end{array}\right.
$$

By Lemma 4.4, we can choose such $L_{\mathbf{n}}$ and $T_{\mathbf{n}}$ inductively. Put $L=1_{r}+$ $\sum_{\mathbf{n} \in \widetilde{\Lambda}} L_{\mathbf{n}} E^{\mathbf{n}}$ and $T=\sum_{\mathbf{n} \in \widetilde{\Lambda}} T_{\mathbf{n}} E^{\mathbf{n}}$. Since $f(\mathbf{n})(H) \neq\left(Q_{0}\right)_{i i}-\left(Q_{0}\right)_{j j}$ implies $\left(T_{\mathbf{n}}\right)_{i j}=0$, we have

$$
\begin{aligned}
{\left[H, T_{i j}\right] } & =\sum_{\mathbf{n} \in \widetilde{\Lambda}}(f(\mathbf{n})(H))\left(T_{\mathbf{n}}\right)_{i j} E^{\mathbf{n}} \\
& =\sum_{\mathbf{n} \in \widetilde{\Lambda}, f(\mathbf{n})(H)=\left(Q_{0}\right)_{i i}-\left(Q_{0}\right)_{j j}}(f(\mathbf{n})(H))\left(T_{\mathbf{n}}\right)_{i j} E^{\mathbf{n}}=\left(\left(Q_{0}\right)_{i i}-\left(Q_{0}\right)_{j j}\right) T_{i j},
\end{aligned}
$$

Hence $L$ and $T$ satisfy the conditions of the lemma.

Proof of Theorem 4.1. We can choose positive integers $\zeta=\left(\zeta_{i}\right) \in \mathbb{Z}_{>0}^{l}$ 
such that

$$
\begin{aligned}
\left\{\alpha \in \Lambda^{++} \mid \alpha\left(\sum_{s} \zeta_{s} H_{s}\right)\right. & \left.=\left(\lambda_{i}-\lambda_{j}\right)\left(\sum_{s} \zeta_{s} H_{s}\right)\right\} \\
& = \begin{cases}\left\{\lambda_{i}-\lambda_{j}\right\} & \left(\lambda_{i}-\lambda_{j} \in \Lambda^{++}\right), \\
\emptyset & \left(\lambda_{i}-\lambda_{j} \notin \Lambda^{++}\right) .\end{cases}
\end{aligned}
$$

The existence of such $\zeta$ is shown by Oshima [Osh84, Lemma 2.3]. Set $X=$ $\sum_{s} \zeta_{s} H_{s}$. Notice that $\left(\lambda_{i}-\lambda_{j}\right)(X) \in\left\{\mu(X) \mid \mu \in \Lambda^{++}\right\}$if and only if $\lambda_{i}-\lambda_{j} \in$ $\Lambda^{++}$. By Lemma 4.5 , there exist $T \in M\left(r,(\mathfrak{n} U(\mathfrak{n}))_{\Lambda}\right)$ and $L \in M\left(r, \widehat{\mathcal{E}}(\mathfrak{n})_{\Lambda}\right)$ such that

$$
\left\{\begin{array}{l}
L \in 1_{r}+M\left(r,(\mathfrak{n} \widehat{\mathcal{E}}(\mathfrak{n}))_{\Lambda}\right), \\
\left(X 1_{r}-\bar{Q}(X)-T\right) L=L\left(X 1_{r}-\bar{Q}(X)-R\right), \\
\text { if } \lambda_{i}-\lambda_{j} \notin \Lambda^{++} \text {then } T_{i j}=0, \\
\text { if } \lambda_{i}-\lambda_{j} \in \Lambda^{++} \text {then }\left[X, T_{i j}\right]=\left(\lambda_{i}-\lambda_{j}\right)(X) T_{i j} .
\end{array}\right.
$$

Let $S \in M\left(N,(\mathfrak{n} U(\mathfrak{n}))_{\Lambda}\right)$ such that $u=\bar{C} w+S u$. Put $C=(1-$ $S)^{-1} \bar{C} L^{-1}, D=L \bar{D}$ and $v=\left(v_{1}, v_{2}, \ldots, v_{r}\right)=D u=L w$. Then $C D u=(1-$ $S)^{-1} \bar{C} L^{-1} L \bar{D} u=(1-S)^{-1} \bar{C} w=u$. Moreover, we have $\left(X 1_{r}-\bar{Q}(X)-T\right) v=0$.

Assume $i<j$. Then $\operatorname{Re} \lambda_{i}-\operatorname{Re} \lambda_{j} \leq 0$. Hence $\lambda_{i}-\lambda_{j} \notin \Lambda^{++}$. So we have $T_{i j}=0$. From this fact and $\left(X 1_{r}-\bar{Q}(X)-T\right) v=0$, we have $\left(X-\lambda_{i}(X)\right)^{r-i+1} v_{i}=0$ (see Remark $4.2(2)$ ).

We construct the map $Q$. Fix a positive integer $k$ such that $1 \leq k \leq l$. We can choose a matrix $R_{k} \in M\left(r,(\mathfrak{n} \widehat{\mathcal{E}}(\mathfrak{n}))_{\Lambda}\right)$ such that $H_{k} w=\left(\bar{Q}\left(H_{k}\right)+R_{k}\right) w$ by Lemma 4.3. Set $T_{k}=H_{k} 1_{r}-\bar{Q}\left(H_{k}\right)-L\left(H_{k} 1_{r}-\bar{Q}\left(H_{k}\right)-R_{k}\right) L^{-1}$. Then we have $\left(H_{k} 1_{r}-\bar{Q}\left(H_{k}\right)-T_{k}\right) v=0$, i.e.,

$$
H_{k} v_{i}-\sum_{j=1}^{r} \bar{Q}\left(H_{k}\right)_{i j} v_{j}-\sum_{j=1}^{r}\left(T_{k}\right)_{i j} v_{j}=0
$$

for each $i=1,2, \ldots, r$. By Corollary 3.9 , we have

$$
H_{k} v_{i}-\sum_{j=1}^{r} \bar{Q}\left(H_{k}\right)_{i j} v_{j}-\sum_{j=1}^{r}\left(T_{k}\right)_{i j}^{\left(X,\left(\lambda_{i}-\lambda_{j}\right)(X)\right)} v_{j}=0 .
$$

Define $T_{k}^{\prime} \in M\left(r,(\mathfrak{n} U(\mathfrak{n}))_{\Lambda}\right)$ by $\left(T_{k}^{\prime}\right)_{i j}=\left(T_{k}\right)_{i j}^{\left(X,\left(\lambda_{i}-\lambda_{j}\right)(X)\right)}$. Since $\left(T_{k}\right)_{i j}^{(\mu)}=0$ for $\mu \notin \Lambda^{++}$, we have 


$$
\begin{aligned}
\left(T_{k}\right)_{i j}^{\left(X,\left(\lambda_{i}-\lambda_{j}\right)(X)\right)} & =\sum_{\mu \in \Lambda^{++}, \mu(X)=\left(\lambda_{i}-\lambda_{j}\right)(X)}\left(T_{k}\right)_{i j}^{(\mu)} \\
& = \begin{cases}\left(T_{k}\right)_{i j}^{\left(\lambda_{i}-\lambda_{j}\right)} & \left(\lambda_{i}-\lambda_{j} \in \Lambda^{++}\right), \\
0 & \left(\lambda_{i}-\lambda_{j} \notin \Lambda^{++}\right)\end{cases}
\end{aligned}
$$

by the condition on $\zeta$. In particular $\left[H,\left(T_{k}^{\prime}\right)_{i j}\right]=\left(\lambda_{i}-\lambda_{j}\right)(H)\left(T_{k}^{\prime}\right)_{i j}$ for all $H \in \mathfrak{a}$.

Put $Q\left(\sum x_{s} H_{s}\right)=\bar{Q}\left(\sum x_{s} H_{s}\right)+\sum x_{s} T_{s}^{\prime}$ for $\left(x_{1}, x_{2}, \ldots, x_{l}\right) \in \mathbb{C}^{l}$. Then we have $(H-Q(H)) v=0$ by (4.1). Recall that we have $T_{i j}=0$ for $i<j$. Hence we have $\left(T_{s}^{\prime}\right)_{i j}=0$ for $i<j$. Moreover, by the condition on $\bar{Q}$, we have $\bar{Q}(H)_{i j}=0$ for $i<j$ and $H \in \mathfrak{a}$. Hence we have $Q(H)_{i j}=0$ for $i<j$ and $H \in \mathfrak{a}$. Therefore $Q$ satisfies the conditions of the theorem.

We apply Theorem 4.1 to a principal series representation. First we define the principal series representation which we will study.

Set $\rho=\sum_{\alpha \in \Sigma^{+}}\left(\operatorname{dim} \mathfrak{g}_{\alpha} / 2\right) \alpha$. From the Iwasawa decomposition $\mathfrak{g}=\mathfrak{k} \oplus \mathfrak{a} \oplus \mathfrak{n}$ we have the decomposition into the direct sum

$$
U(\mathfrak{g})=\mathfrak{n} U(\mathfrak{a} \oplus \mathfrak{n}) \oplus U(\mathfrak{a}) \oplus U(\mathfrak{g}) \mathfrak{k} .
$$

Let $\chi_{1}$ be the projection of $U(\mathfrak{g})$ to $U(\mathfrak{a})$ with respect to this decomposition and $\chi_{2}$ an algebra automorphism of $U(\mathfrak{a})$ defined by $\chi_{2}(H)=H-\rho(H)$ for $H \in \mathfrak{a}$. We define $\chi: U(\mathfrak{g})^{\mathfrak{k}} \rightarrow U(\mathfrak{a})$ by $\chi=\chi_{2} \circ \chi_{1}$ where $U(\mathfrak{g})^{\mathfrak{k}}=\{u \in U(\mathfrak{g}) \mid$ $X u=u X$ for all $X \in \mathfrak{k}\}$. Let $U(\mathfrak{a})^{W}$ be a subalgebra of $U(\mathfrak{a})$ consisting of $W$ invariant elements. It is known that the image of $U(\mathfrak{g})^{\mathfrak{k}}$ under $\chi$ is contained in $U(\mathfrak{a})^{W}$ and induces an isomorphism of algebra $U(\mathfrak{g})^{\mathfrak{k}} /\left(U(\mathfrak{g})^{\mathfrak{k}} \cap U(\mathfrak{g}) \mathfrak{k}\right) \rightarrow U(\mathfrak{a})^{W}$. For details, see Helgason [Hel62, Chapter X, §6.3].

Fix $\lambda \in \mathfrak{a}^{*}$. We can define an algebra homomorphism $U(\mathfrak{a}) \rightarrow \mathbb{C}$ by $H \mapsto \lambda(H)$ for $H \in \mathfrak{a}$. We denote this map by the same letter $\lambda$. Put $\chi_{\lambda}=\lambda \circ \chi$. Define the $U(\mathfrak{g})$-module $I(\lambda)$ by

$$
I(\lambda)=U(\mathfrak{g}) /\left(U(\mathfrak{g}) \operatorname{Ker} \chi_{\lambda}+U(\mathfrak{g}) \mathfrak{k}\right) .
$$

By a result of Kostant [Kos75, Theorem 2.10.3], $I(\lambda)$ is isomorphic to the principal series representation generated by the trivial $K$-type with an infinitesimal character $\lambda$ (see p. 1194). Set $u_{\lambda}=1 \bmod \left(U(\mathfrak{g}) \operatorname{Ker} \chi_{\lambda}+U(\mathfrak{g}) \mathfrak{k}\right) \in I(\lambda)$ and $I(\lambda)_{0}=U(\mathfrak{g})_{2} \mathcal{P} u_{\lambda}$. Before applying Theorem 4.1 to $I(\lambda)_{0}$, we give some lemmas.

Lemma 4.6. Let $u \in U(\mathfrak{g})_{\mu}$ where $\mu \in \mathfrak{a}^{*}$. Then there exists an element $x \in U(\mathfrak{g}) \mathfrak{k}$ such that $u+x \in U(\mathfrak{a} \oplus \mathfrak{n})_{\mu+2 \mathcal{P}}$. 
Proof. In general, for a Lie algebra $\mathfrak{c}$, let $\left\{U_{n}(\mathfrak{c})\right\}_{n \in \mathbb{Z}_{\geq 0}}$ be the standard filtration of $U(\mathfrak{c})$. Let $n$ be the smallest integer such that $u \in U_{n}(\mathfrak{g})$. We will prove the existence of $x$ by induction on $n$.

If $n=0$ then the lemma is obvious. Assume $n>0$. Set $\overline{\mathfrak{n}}=\theta(\mathfrak{n})$. First assume that $u \in U_{n}(\overline{\mathfrak{n}})_{\mu}$. We may assume that there exist a restricted root $\alpha \in \Sigma^{+}$, an element $u_{0} \in U_{n-1}(\overline{\mathfrak{n}})_{\mu+\alpha}$ and a vector $E_{-\alpha} \in \mathfrak{g}_{-\alpha}$ such that $u=u_{0} E_{-\alpha}$. Set $E_{\alpha}=\theta\left(E_{-\alpha}\right), u_{1}=u_{0} E_{\alpha}, u_{2}=E_{\alpha} u_{0}$ and $u_{3}=u_{1}-u_{2}$. Then $u+u_{2}+u_{3}=u+u_{1} \in U(\mathfrak{g}) \mathfrak{k}, u_{1}, u_{2} \in U(\mathfrak{g})_{\mu+2 \alpha}$ and $u_{3} \in U_{n-1}(\mathfrak{g})_{\mu+2 \alpha}$. Using the induction hypothesis we can choose an element $u_{5} \in U(\mathfrak{a} \oplus \mathfrak{n})_{\mu+2 \mathcal{P}}$ such that $u_{3}-u_{5} \in U(\mathfrak{g}) \mathfrak{k}$. Again by the induction hypothesis we can choose an element $u_{6} \in U(\mathfrak{a} \oplus \mathfrak{n})_{\mu+\alpha+2 \mathcal{P}}$ such that $u_{0}-u_{6} \in U(\mathfrak{g}) \mathfrak{k}$. Then $u+u_{5}+E_{\alpha} u_{6} \in U(\mathfrak{g}) \mathfrak{k}$, $u_{5} \in U(\mathfrak{a} \oplus \mathfrak{n})_{\mu+2 \mathcal{P}}$ and $E_{\alpha} u_{6} \in U(\mathfrak{a} \oplus \mathfrak{n})_{\mu+2 \alpha+2 \mathcal{P}}$.

Now assume that $u \in U_{n}(\mathfrak{g})_{\mu}$. Since $U_{n}(\mathfrak{g})_{\mu}=\sum_{k=0}^{n} \bigoplus_{\mu_{1}} U_{n-k}(\mathfrak{a} \oplus \mathfrak{n} \oplus$ $\mathfrak{m})_{\mu-\mu_{1}} \otimes U_{k}(\overline{\mathfrak{n}})_{\mu_{1}}$, we may assume that $u=u^{\prime} u^{\prime \prime}$ where $u^{\prime} \in U_{n-k}(\mathfrak{a} \oplus \mathfrak{n} \oplus \mathfrak{m})_{\mu-\mu_{1}}$ and $u^{\prime \prime} \in U_{k}(\overline{\mathfrak{n}})_{\mu_{1}}$. Take $x \in U(\mathfrak{g})^{\mathfrak{k}}$ such that $x+u^{\prime \prime} \in U(\mathfrak{a} \oplus \mathfrak{n})_{\mu_{1}+2 \mathcal{P}}$ and put $x^{\prime}=u^{\prime} x \in U(\mathfrak{g})^{\mathfrak{k}}$. Then we have $u+x^{\prime} \in U(\mathfrak{a} \oplus \mathfrak{n} \oplus \mathfrak{m})_{\mu+2 \mathcal{P}}=U(\mathfrak{a} \oplus \mathfrak{n})_{\mu+2 \mathcal{P}} \oplus$ $U(\mathfrak{a} \oplus \mathfrak{n})_{\mu+2 \mathcal{P}} \mathfrak{m}$. Take $x^{\prime \prime} \in U(\mathfrak{a} \oplus \mathfrak{n})_{\mu+2 \mathcal{P}} \mathfrak{m}$ such that $u+x^{\prime}-x^{\prime \prime} \in U(\mathfrak{a} \oplus \mathfrak{n})_{\mu+2 \mathcal{P}}$. Since $\mathfrak{m} \subset \mathfrak{k}, x^{\prime}-x^{\prime \prime} \in U(\mathfrak{g})_{\mathfrak{k}}+U(\mathfrak{a} \oplus \mathfrak{n})_{\mu+2 \mathcal{P}} \mathfrak{m}=U(\mathfrak{g})^{\mathfrak{k}}$. Hence we have the lemma.

Lemma 4.7. The following formulae hold.

(1) $U(\mathfrak{g})^{\mathfrak{k}} \subset U(\mathfrak{g})_{2 \mathcal{P}}$. In particular, we have $\operatorname{Ker} \chi_{\lambda} \subset U(\mathfrak{g})_{2 \mathcal{P}}$.

(2) $U(\mathfrak{a} \oplus \mathfrak{n}) \cap\left(\operatorname{Ker} \chi_{\lambda}+U(\mathfrak{g}) \mathfrak{k}\right) \subset U(\mathfrak{a} \oplus \mathfrak{n})_{2 \mathcal{P}} \cap\left(\operatorname{Ker} \chi_{\lambda}+U(\mathfrak{g}) \mathfrak{k}\right)$.

(3) $U(\mathfrak{a} \oplus \mathfrak{n}) \cap\left(U(\mathfrak{a} \oplus \mathfrak{n}) \operatorname{Ker} \chi_{\lambda}+U(\mathfrak{g}) \mathfrak{k}\right) \subset U(\mathfrak{a} \oplus \mathfrak{n})\left(U(\mathfrak{a} \oplus \mathfrak{n}) \cap\left(\operatorname{Ker} \chi_{\lambda}+U(\mathfrak{g}) \mathfrak{k}\right)\right)$.

(4) $U(\mathfrak{a} \oplus \mathfrak{n}) \cap\left(U(\mathfrak{g}) \operatorname{Ker} \chi_{\lambda}+U(\mathfrak{g}) \mathfrak{k}\right)=U(\mathfrak{a} \oplus \mathfrak{n})\left(\left(U(\mathfrak{g}) \operatorname{Ker} \chi_{\lambda}+U(\mathfrak{g}) \mathfrak{k}\right) \cap U(\mathfrak{a} \oplus\right.$ $\left.\mathfrak{n})_{2 \mathcal{P}}\right)$.

Proof. (1) Take a connected Lie group $G$ such that Lie algebra of $G$ is $\mathfrak{g}_{0}$ and $G$ has a complexification. Let $K$ be its maximal compact subgroup such that $\operatorname{Lie}(K)=\mathfrak{k}_{0}$. Since $K$ is connected, $U(\mathfrak{g})^{\mathfrak{k}}=U(\mathfrak{g})^{K}=\{u \in U(\mathfrak{g}) \mid$ $\operatorname{Ad}(k) u=u$ for all $k \in K\}$. Fix a maximal abelian subspace $\mathfrak{t}_{0}$ of $\mathfrak{m}_{0}$. Let $K_{\text {split }}$ and $A_{\text {split }}$ be the analytic subgroups with Lie algebras given as the intersections of $\mathfrak{k}_{0}$ and $\mathfrak{a}_{0}$ with $\left[Z_{\mathfrak{g}_{0}}\left(\mathfrak{t}_{0}\right), Z_{\mathfrak{g}_{0}}\left(\mathfrak{t}_{0}\right)\right]$ where $Z_{\mathfrak{g}_{0}}\left(\mathfrak{t}_{0}\right)$ is the centralizer of $\mathfrak{t}_{0}$ in $\mathfrak{g}_{0}$. Let $F$ be the centralizer of $A_{\text {split }}$ in $K_{\text {split }}$. Since $F \subset K$, we have $U(\mathfrak{g})^{K} \subset$ $U(\mathfrak{g})^{F}$. On the other hand, we have $U(\mathfrak{g})^{F} \subset U(\mathfrak{g})_{2 \mathcal{P}}$ (See Knapp [Kna02, Theorem 7.55] and Lepowsky [Lep75, Proposition 6.1, Proposition 6.4]). Hence (1) follows. 
(2) Let $u \in \operatorname{Ker} \chi_{\lambda}$ and $x \in U(\mathfrak{g}) \mathfrak{k}$ such that $u+x \in U(\mathfrak{a} \oplus \mathfrak{n})$. We can write $u=\sum_{\mu} u_{\mu}$ where $u_{\mu} \in U(\mathfrak{g})_{\mu}$. By (1), we have $u_{\mu}=0$ for $\mu \notin 2 \mathcal{P}$. Let $\mu \in 2 \mathcal{P}$. By Lemma 4.6, there exists an element $y_{\mu} \in U(\mathfrak{g}) \mathfrak{k}$ such that $u_{\mu}+y_{\mu} \in U(\mathfrak{a} \oplus \mathfrak{n})_{\mu+2 \mathcal{P}}=U(\mathfrak{a} \oplus \mathfrak{n})_{2 \mathcal{P}}$. Put $y=\sum_{\mu} y_{\mu}$. Then $u+y \in U(\mathfrak{a} \oplus \mathfrak{n})_{2 \mathcal{P}}$. Since $u+y \in U(\mathfrak{a} \oplus \mathfrak{n})$ and $x, y \in U(\mathfrak{g}) \mathfrak{k}$ we have $y=x$ by the Poincaré-BirkhoffWitt theorem. Hence we have $u+x=u+y \in U(\mathfrak{a} \oplus \mathfrak{n})_{2} \mathcal{P}$.

(3) Let $\sum_{i} x_{i} u_{i} \in U(\mathfrak{a} \oplus \mathfrak{n}) \operatorname{Ker} \chi_{\lambda}$ where $x_{i} \in U(\mathfrak{a} \oplus \mathfrak{n})$ and $u_{i} \in \operatorname{Ker} \chi_{\lambda}$. We write $u_{i}=\sum_{j} z_{j}^{(i)} v_{j}^{(i)}$ where $z_{j}^{(i)} \in U(\mathfrak{a} \oplus \mathfrak{n})$ and $v_{j}^{(i)} \in U(\mathfrak{k})$. Let $y \in U(\mathfrak{g}) \mathfrak{k}$ and assume $\sum_{i} x_{i} u_{i}+y \in U(\mathfrak{a} \oplus \mathfrak{n})$. By the Poincaré-Birkhoff-Witt theorem, $\sum_{i} x_{i} u_{i}+y=\sum_{i, j} x_{i} z_{j}^{(i)} v_{j, 0}^{(i)}$ where $v_{j, 0}^{(i)}$ is the constant term of $v_{j}^{(i)}$. Since $u_{i}+\sum_{j} z_{j}^{(i)}\left(v_{j, 0}^{(i)}-v_{j}^{(i)}\right)=\sum_{j} z_{j}^{(i)} v_{j, 0}^{(i)} \in U(\mathfrak{a} \oplus \mathfrak{n})$, we have $\sum_{i} x_{i} u_{i}+y=$ $\sum_{i} x_{i}\left(u_{i}+\sum_{j} z_{j}^{(i)}\left(v_{j, 0}^{(i)}-v_{j}^{(i)}\right)\right) \in U(\mathfrak{a} \oplus \mathfrak{n})\left(U(\mathfrak{a} \oplus \mathfrak{n}) \cap\left(\operatorname{Ker} \chi_{\lambda}+U(\mathfrak{g}) \mathfrak{k}\right)\right)$.

(4) Since Ker $\chi_{\lambda} \subset U(\mathfrak{g})^{\mathfrak{k}}$, we have

$U(\mathfrak{g}) \operatorname{Ker} \chi_{\lambda}+U(\mathfrak{g}) \mathfrak{k}=U(\mathfrak{a} \oplus \mathfrak{n})\left(\operatorname{Ker} \chi_{\lambda}\right) U(\mathfrak{k})+U(\mathfrak{g}) \mathfrak{k}=U(\mathfrak{a} \oplus \mathfrak{n}) \operatorname{Ker} \chi_{\lambda}+U(\mathfrak{g}) \mathfrak{k}$.

By (2) and (3), we have

$$
\begin{aligned}
U(\mathfrak{a} \oplus \mathfrak{n}) \cap\left(U(\mathfrak{g}) \operatorname{Ker} \chi_{\lambda}\right. & +U(\mathfrak{g}) \mathfrak{k}) \\
& =U(\mathfrak{a} \oplus \mathfrak{n}) \cap\left(U(\mathfrak{a} \oplus \mathfrak{n}) \operatorname{Ker} \chi_{\lambda}+U(\mathfrak{g}) \mathfrak{k}\right) \\
& \subset U(\mathfrak{a} \oplus \mathfrak{n})\left(U(\mathfrak{a} \oplus \mathfrak{n})_{2 \mathcal{P}} \cap\left(\operatorname{Ker} \chi_{\lambda}+U(\mathfrak{g}) \mathfrak{k}\right)\right) \\
& \subset U(\mathfrak{a} \oplus \mathfrak{n})\left(\left(U(\mathfrak{g}) \operatorname{Ker} \chi_{\lambda}+U(\mathfrak{g}) \mathfrak{k}\right) \cap U(\mathfrak{a} \oplus \mathfrak{n})_{2 \mathcal{P}}\right) .
\end{aligned}
$$

This implies (4).

\section{Lemma 4.8.}

(1) We have $I(\lambda)_{0}=U(\mathfrak{a} \oplus \mathfrak{n})_{2 \mathcal{P}} u_{\lambda}$.

(2) The map $p \otimes u \mapsto p u$ induces an isomorphism $U(\mathfrak{n}) \otimes_{U(\mathfrak{n})_{2 \mathcal{P}}} I(\lambda)_{0} \simeq I(\lambda)$.

Proof. (1) Since $\mathfrak{k} u_{\lambda}=0$, this is obvious from Lemma 4.6.

(2) Let $I=U(\mathfrak{a} \oplus \mathfrak{n}) \cap\left(U(\mathfrak{g}) \operatorname{Ker} \chi_{\lambda}+U(\mathfrak{g}) \mathfrak{k}\right)$. We have $U(\mathfrak{a} \oplus \mathfrak{n}) \otimes_{U(\mathfrak{a} \oplus \mathfrak{n})_{2 \mathcal{P}}} \mathbb{X}=$ $U(\mathfrak{n}) \otimes_{U(\mathfrak{n})_{2 \mathcal{P}}} \mathbb{X}$ for any $U(\mathfrak{a} \oplus \mathfrak{n})_{2 \mathcal{P}}$-module $\mathbb{X}$ since $U(\mathfrak{a} \oplus \mathfrak{n})_{2 \mathcal{P}}=U(\mathfrak{a}) \otimes U(\mathfrak{n})_{2 \mathcal{P}}$.

By (1), we have $I(\lambda)_{0}=U(\mathfrak{a} \oplus \mathfrak{n})_{2 \mathcal{P}} /\left(I \cap U(\mathfrak{a} \oplus \mathfrak{n})_{2 \mathcal{P}}\right)$. Hence

$$
\begin{aligned}
U(\mathfrak{n}) \otimes_{U(\mathfrak{n})_{2 \mathcal{P}}} I(\lambda)_{0} & =U(\mathfrak{a} \oplus \mathfrak{n}) \otimes_{U(\mathfrak{a} \oplus \mathfrak{n})_{2 \mathcal{P}}} I(\lambda)_{0} \\
& =U(\mathfrak{a} \oplus \mathfrak{n}) \otimes_{U(\mathfrak{a} \oplus \mathfrak{n})_{2 \mathcal{P}}}\left(U(\mathfrak{a} \oplus \mathfrak{n})_{2 \mathcal{P}} /\left(I \cap U(\mathfrak{a} \oplus \mathfrak{n})_{2 \mathcal{P}}\right)\right) \\
& =U(\mathfrak{a} \oplus \mathfrak{n}) /\left(U(\mathfrak{a} \oplus \mathfrak{n})\left(I \cap U(\mathfrak{a} \oplus \mathfrak{n})_{2 \mathcal{P}}\right)\right) \\
& =U(\mathfrak{a} \oplus \mathfrak{n}) / I \\
& =I(\lambda)
\end{aligned}
$$


by Lemma 4.7 (4).

Lemma 4.9. Let $\left\{U_{n}(\mathfrak{n})\right\}_{n \in \mathbb{Z}_{>0}}$ be the standard filtration of $U(\mathfrak{n})$ and $U_{n}(\mathfrak{n})_{2 \mathcal{P}}=U_{n}(\mathfrak{n}) \cap U(\mathfrak{n})_{2 \mathcal{P}}$. Set $U_{-1}(\mathfrak{n})=U_{-1}(\mathfrak{n})_{2 \mathcal{P}}=0, R=\operatorname{Gr} U(\mathfrak{n})_{2 \mathcal{P}}=$ $\bigoplus_{n} U_{n}(\mathfrak{n})_{2 \mathcal{P}} / U_{n-1}(\mathfrak{n})_{2 \mathcal{P}}$ and $R^{\prime}=\operatorname{Gr} U(\mathfrak{n})=\bigoplus_{n} U_{n}(\mathfrak{n}) / U_{n-1}(\mathfrak{n})$.

(1) $R^{\prime}$ is a finitely generated $R$-module.

(2) $U(\mathfrak{n})$ is a finitely generated $U(\mathfrak{n})_{2 \mathcal{P}}$-module.

(3) $U(\mathfrak{n})_{2 \mathcal{P}}$ is right and left Noetherian.

(4) $I(\lambda)_{0}$ is a finitely generated $U(\mathfrak{n})_{2 \mathcal{P}}$-module.

Proof. (1) Let $\Gamma=\left\{E^{\varepsilon} \mid \varepsilon \in\{0,1\}^{m}\right\}$. We denote the principal symbol of $u \in U(\mathfrak{n})$ by $\sigma(u) \in R^{\prime}$. Notice that if $u \in U(\mathfrak{n})_{2 \mathcal{P}}$ then $\sigma(u)$ is the principal symbol of $u$ as an element of $U(\mathfrak{n})_{2} \mathcal{P}$.

We will prove that $\{\sigma(E) \mid E \in \Gamma\}$ generates $R^{\prime}$ as an $R$-module. Let $x \in R^{\prime}$. We may assume that $x$ is homogeneous, so there exists an element $u \in U(\mathfrak{n})$ such that $x=\sigma(u)$. Moreover we may assume that there exist nonnegative integers $\mathbf{p}=\left(p_{1}, p_{2}, \ldots, p_{m}\right)$ such that $u=E^{\mathbf{p}}$. Choose $\varepsilon_{i} \in\{0,1\}$ such that $\varepsilon_{i} \equiv p_{i}(\bmod 2)$. Set $q_{i}=\left(p_{i}-\varepsilon_{i}\right) / 2 \in \mathbb{Z}_{\geq 0}, \varepsilon=\left(\varepsilon_{1}, \varepsilon_{2}, \ldots, \varepsilon_{m}\right)$ and $\mathbf{q}=\left(q_{1}, q_{2}, \ldots, q_{m}\right)$. Then we have $x=\sigma\left(E^{\mathbf{p}}\right)=\sigma\left(E^{2 \mathbf{q}}\right) \sigma\left(E^{\varepsilon}\right)$. Since $\sigma\left(E^{2 \mathbf{q}}\right) \in R$, this implies that $\{\sigma(E) \mid E \in \Gamma\}$ generates $R^{\prime}$ as an $R$-module.

(2) This is a direct consequence of (1).

(3) By the Poincaré-Birkhoff-Witt theorem, $R^{\prime}$ is isomorphic to a polynomial ring. In particular $R^{\prime}$ is Noetherian. By the theorem of Eakin-Nagata and (1), we have $R$ is Noetherian. This implies (3).

(4) Since $I(\lambda)$ is a finite-length $(\mathfrak{g}, K)$-module, $I(\lambda)$ is a finitely generated $U(\mathfrak{n})$-module by a theorem of Casselman-Osborne [CO78, 2.3 Theorem]. Since $U(\mathfrak{n})$ is a finitely generated $U(\mathfrak{n})_{2 \mathcal{P}}$-module, $I(\lambda)$ is a finitely generated $U(\mathfrak{n})_{2} \mathcal{P}^{-}$ module. Hence $I(\lambda)_{0}$ is a finitely generated $U(\mathfrak{n})_{2 \mathcal{P}}$-module by $(3)$.

We enumerate $W=\left\{w_{1}, w_{2}, \ldots, w_{r}\right\}$ such that $\operatorname{Re} w_{1} \lambda \geq \operatorname{Re} w_{2} \lambda \geq \cdots \geq$ $\operatorname{Re} w_{r} \lambda$.

Theorem 4.10. There exist matrices $A \in M\left(1, r, \widehat{\mathcal{E}}(\mathfrak{n})_{2 \mathcal{P}}\right)$ and $B \in$ $M\left(r, 1, \widehat{\mathcal{E}}(\mathfrak{a} \oplus \mathfrak{n}, \mathfrak{n})_{2 \mathcal{P}}\right)$ such that $v_{\lambda}=B u_{\lambda} \in\left(\widehat{\mathcal{E}}(\mathfrak{g}, \mathfrak{n}) \otimes_{U(\mathfrak{g})} I(\lambda)\right)^{r}$ satisfies the following conditions: 
- There exists a linear map $Q: \mathfrak{a} \rightarrow M\left(r, U(\mathfrak{n})_{2} \mathcal{P}\right)$ such that

$$
\left\{\begin{array}{l}
H v_{\lambda}=Q(H) v_{\lambda} \text { for all } H \in \mathfrak{a}, \\
Q(H)_{i i}=\left(\rho+w_{i} \lambda\right)(H) \text { for all } H \in \mathfrak{a}, \\
\text { if } w_{i} \lambda-w_{j} \lambda \notin 2 \mathcal{P}^{+} \text {then } Q(H)_{i j}=0 \text { for all } H \in \mathfrak{a}, \\
\text { if } w_{i} \lambda-w_{j} \lambda \in 2 \mathcal{P}^{+} \text {then }\left[H^{\prime}, Q(H)_{i j}\right] \\
\quad=\left(w_{i} \lambda-w_{j} \lambda\right)\left(H^{\prime}\right) Q(H)_{i j} \text { for all } H, H^{\prime} \in \mathfrak{a}, \\
\text { if } i>j \text { then } Q(H)_{i j}=0 \text { for all } H \in \mathfrak{a} .
\end{array}\right.
$$

- We have $u_{\lambda}=A v_{\lambda}$.

- Let $\left(v_{1}, v_{2}, \ldots, v_{r}\right)=v_{\lambda}$. Then $\left\{v_{i}(\bmod \mathfrak{n} I(\lambda))\right\}$ is a basis of $I(\lambda) / \mathfrak{n} I(\lambda)$.

Proof. Let $u_{1}, u_{2}, \ldots, u_{N}$ be generators of $I(\lambda)_{0}$ as a $U(\mathfrak{n})_{2 \mathcal{P}}$-module. These are also generators of $I(\lambda)$ as a $U(\mathfrak{n})$-module by Lemma 4.8. We choose matrices $E={ }^{t}\left(E_{1}, E_{2}, \ldots, E_{N}\right) \in M\left(N, 1, U(\mathfrak{a} \oplus \mathfrak{n})_{2 \mathcal{P}}\right)$ and $F=\left(F_{1}, F_{2}, \ldots\right.$, $\left.F_{N}\right) \in M\left(1, N, U(\mathfrak{n})_{2 \mathcal{P}}\right)$ such that ${ }^{t}\left(u_{1}, u_{2}, \ldots, u_{N}\right)=E u_{\lambda}$ and $u_{\lambda}=F^{t}\left(u_{1}, u_{2}\right.$, $\left.\ldots, u_{N}\right)$.

Notice that $U(\mathfrak{n})_{2 \mathcal{P}}+\mathfrak{n} U(\mathfrak{n})=U(\mathfrak{n})$. By Lemma 4.8,

$$
\begin{aligned}
I(\lambda) / \mathfrak{n} I(\lambda) & =(U(\mathfrak{n}) / \mathfrak{n} U(\mathfrak{n})) \otimes_{U(\mathfrak{n})} I(\lambda) \\
& =(U(\mathfrak{n}) / \mathfrak{n} U(\mathfrak{n})) \otimes_{U(\mathfrak{n})} U(\mathfrak{n}) \otimes_{U(\mathfrak{n})_{2 \mathcal{P}}} I(\lambda)_{0} \\
& =(U(\mathfrak{n}) / \mathfrak{n} U(\mathfrak{n})) \otimes_{U(\mathfrak{n})_{2 \mathcal{P}}} I(\lambda)_{0} \\
& =\left(\left(U(\mathfrak{n})_{2 \mathcal{P}}+\mathfrak{n} U(\mathfrak{n})\right) / \mathfrak{n} U(\mathfrak{n})\right) \otimes_{U(\mathfrak{n})_{2 \mathcal{P}}} I(\lambda)_{0} \\
& =\left(U(\mathfrak{n})_{2 \mathcal{P}} /\left(\mathfrak{n} U(\mathfrak{n}) \cap U(\mathfrak{n})_{2 \mathcal{P}}\right)\right) \otimes_{U(\mathfrak{n})_{2 \mathcal{P}}} I(\lambda)_{0} \\
& =\left(U(\mathfrak{n})_{2 \mathcal{P}} /(\mathfrak{n} U(\mathfrak{n}))_{2 \mathcal{P}}\right) \otimes_{U(\mathfrak{n})_{2 \mathcal{P}}} I(\lambda)_{0} \\
& =I(\lambda)_{0} /(\mathfrak{n} U(\mathfrak{n}))_{2 \mathcal{P}} I(\lambda)_{0} .
\end{aligned}
$$

On the other hand,

$$
\begin{aligned}
I(\lambda) / \mathfrak{n} I(\lambda) & =U(\mathfrak{g}) /\left(\mathfrak{n} U(\mathfrak{g})+U(\mathfrak{g}) \operatorname{Ker} \chi_{\lambda}+U(\mathfrak{g}) \mathfrak{k}\right) \\
& =(\mathfrak{n} U(\mathfrak{g})+U(\mathfrak{a})+U(\mathfrak{g}) \mathfrak{k}) /\left(\mathfrak{n} U(\mathfrak{g})+U(\mathfrak{g}) \operatorname{Ker} \chi_{\lambda}+U(\mathfrak{g}) \mathfrak{k}\right) \\
& =U(\mathfrak{a}) /\left(\left(\mathfrak{n} U(\mathfrak{g})+U(\mathfrak{g}) \operatorname{Ker} \chi_{\lambda}+U(\mathfrak{g}) \mathfrak{k}\right) \cap U(\mathfrak{a})\right) .
\end{aligned}
$$

Since $\chi$ induces an algebra isomorphism $U(\mathfrak{g})^{\mathfrak{k}} /\left(U(\mathfrak{g})^{\mathfrak{k}} \cap U(\mathfrak{g}) \mathfrak{k}\right) \rightarrow U(\mathfrak{a})^{W}$, $\chi_{1}$ also induces an isomorphism Ker $\chi_{\lambda} /\left(\operatorname{Ker} \chi_{\lambda} \cap U(\mathfrak{g}) \mathfrak{k}\right) \rightarrow \sum_{p \in U(\mathfrak{a})^{W}}\left(\chi_{2}^{-1}(p)-\right.$ $\lambda(p))$. Hence by the definition of $\chi_{1}$, we have

$$
\left(\mathfrak{n} U(\mathfrak{g})+\operatorname{Ker} \chi_{\lambda}+U(\mathfrak{g}) \mathfrak{k}\right)=\mathfrak{n} U(\mathfrak{g})+\left(\sum_{p \in U(\mathfrak{a})^{W}}\left(\chi_{2}^{-1}(p)-\lambda(p)\right)\right)+U(\mathfrak{g}) \mathfrak{k} .
$$


By the Poincaré-Birkhoff-Witt theorem, we have

$$
\left(\mathfrak{n} U(\mathfrak{g})+\operatorname{Ker} \chi_{\lambda}+U(\mathfrak{g}) \mathfrak{k}\right) \cap U(\mathfrak{a})=\sum_{p \in U(\mathfrak{a})^{W}}\left(\chi_{2}^{-1}(p)-\lambda(p)\right) .
$$

Since $\operatorname{Ker} \chi_{\lambda} \subset U(\mathfrak{g})^{\mathfrak{k}}$ and $\mathfrak{a}$ normalizes $\mathfrak{n}$, we have $U(\mathfrak{g}) \operatorname{Ker} \chi_{\lambda}=(\mathfrak{n} U(\mathfrak{g})+$ $U(\mathfrak{a})+U(\mathfrak{g}) \mathfrak{k}) \operatorname{Ker} \chi_{\lambda} \subset \mathfrak{n} U(\mathfrak{g})+U(\mathfrak{a}) \operatorname{Ker} \chi_{\lambda}+U(\mathfrak{g}) \mathfrak{k}=U(\mathfrak{a})\left(\mathfrak{n} U(\mathfrak{g})+\operatorname{Ker} \chi_{\lambda}+\right.$ $U(\mathfrak{g}) \mathfrak{k})$. Hence we have

$$
\left(\mathfrak{n} U(\mathfrak{g})+U(\mathfrak{g}) \operatorname{Ker} \chi_{\lambda}+U(\mathfrak{g}) \mathfrak{k}\right) \cap U(\mathfrak{a})=\sum_{p \in U(\mathfrak{a})^{W}} U(\mathfrak{a})\left(\chi_{2}^{-1}(p)-\lambda(p)\right)
$$

The set of eigenvalues of $H \in \mathfrak{a}$ on $U(\mathfrak{a}) /\left(\sum_{p \in U(\mathfrak{a}){ }_{W}} U(\mathfrak{a})\left(\chi_{2}^{-1}(p)-\lambda(p)\right)\right)$ is $\{(\rho+w \lambda)(H) \mid w \in W\}$ with multiplicities [Osh88, Proposition 2.8].

Put $\mathbb{X}=I(\lambda)_{0}, \Lambda=2 \mathcal{P}$ and apply Theorem 4.1. We take matrices $C \in M\left(N, r, \widehat{\mathcal{E}}(\mathfrak{n})_{2 \mathcal{P}}\right)$ and $D \in M\left(r, N, \widehat{\mathcal{E}}(\mathfrak{n})_{2 \mathcal{P}}\right)$ such that the conditions of Theorem 4.1 hold. Put $A=F C, B=C E$. Then $A$ and $B$ satisfy the conditions of the theorem (for the second condition on $Q$, see Remark $4.2(1)$ ).

Remark 4.11. For all $H \in \mathfrak{a}$ we have $\left(H-\left(w_{i} \lambda+\rho\right)(H)\right)^{r-i+1} v_{i}=0$. See Remark 4.2 (2).

\section{$\S 5 . \quad$ Structure of Jacquet Modules (Regular Case)}

In this section we assume that $\lambda$ is regular, i.e., $w \lambda \neq \lambda$ for all $w \in$ $W \backslash\{e\}$. Let $r=\# W$ and $v_{\lambda}=\left(v_{1}, v_{2}, \ldots, v_{r}\right) \in\left(\widehat{\mathcal{E}}(\mathfrak{g}, \mathfrak{n}) \otimes_{U(\mathfrak{g})} I(\lambda)\right)^{r}$ as in Theorem 4.10. Set $\mathcal{W}(i)=\left\{j \mid w_{i} \lambda-w_{j} \lambda \in 2 \mathcal{P}^{+}\right\}$for each $i=1,2, \ldots, r$.

Proposition 5.1. The vector $v_{i}$ is a lowest weight vector of $J(I(\lambda)) /$ $\sum_{j \in \mathcal{W}(i)} U(\mathfrak{g}) v_{j}$, i.e., we have $X v_{i} \in \sum_{j \in \mathcal{W}(i)} U(\mathfrak{g}) v_{j}$ for all $X \in \theta(\mathfrak{n}) \oplus \mathfrak{m}$.

Let $A={ }^{t}\left(A^{(1)}, A^{(2)}, \ldots, A^{(r)}\right)$ be as in Theorem 4.10 and $\bar{A}={ }^{t}\left(\overline{A^{(1)}}, \overline{A^{(2)}}\right.$, $\left.\ldots, \overline{A^{(r)}}\right)$ an element of $M(r, 1, \mathbb{C})$ such that $A^{(i)}-\overline{A^{(i)}} \in \mathfrak{n} \widehat{\mathcal{E}}(\mathfrak{n})$.

Lemma 5.2. We have $\overline{A^{(i)}} \neq 0$ for each $i=1,2, \ldots, r$.

Proof. Put $\overline{I(\lambda)}=I(\lambda) / \mathfrak{n} I(\lambda) \simeq \widehat{J}(I(\lambda)) / \mathfrak{n} \widehat{J}(I(\lambda))$. Since $u_{\lambda}$ generates $I(\lambda)$, there exists $\bar{B}=\left(\overline{B^{(1)}}, \overline{B^{(2)}}, \ldots, \overline{B^{(r)}}\right) \in U(\mathfrak{g})^{r}$ such that $v_{i}-\overline{B^{(i)}} u_{\lambda} \in$ $\mathfrak{n} \widehat{J}(I(\lambda))$. From $\mathfrak{k} u_{\lambda}=0$ and the Iwasawa decomposition, we may assume $\overline{B^{(i)}} \in U(\mathfrak{a})$.

Put $\overline{u_{\lambda}}=u_{\lambda}(\bmod \mathfrak{n} \widehat{J}(I(\lambda)))$ and $\overline{v_{i}}=v_{i}(\bmod \mathfrak{n} I(\lambda))$. Then we have $\overline{v_{i}}=\sum_{j} \overline{A^{(j)}} \overline{B^{(i)}} \overline{v_{j}}$. 
We can choose $H \in \mathfrak{a}$ such that $\left(\rho+w_{i} \lambda\right)(H) \neq\left(\rho+w_{j} \lambda\right)(H)$ for all $i \neq j$ since $\lambda$ is regular. Then we have $\left(H-\left(\rho+w_{i} \lambda\right)(H)\right)^{r-i+1} v_{i}=0$ (Remark 4.11). Since $\overline{v_{i}}$ is a basis of $\overline{I(\lambda)}$, we have $\left(H-\left(\rho+w_{i} \lambda\right)(H)\right) \overline{v_{i}}=0$. Hence for all $i=1, \ldots, r$ there exists a polynomial $f_{i}$ such that $f_{i}(H)$ is a projection $\overline{I(\lambda)}=\bigoplus_{j} \mathbb{C} \overline{v_{j}} \rightarrow \mathbb{C} \overline{v_{i}}$. Since $\mathfrak{a}$ is abelian, we have $v_{i}=f_{i}(H) v_{i}=$ $\sum_{j} \overline{A^{(j)}} \overline{B^{(i)}} f_{i}(H) \overline{v_{j}}=\overline{A^{(i)}} \overline{B^{(i)}} \overline{v_{i}}$. This implies $\overline{A^{(i)}} \neq 0$.

Proof of Proposition 5.1. Put $f(\mathbf{n})=\sum_{i} \mathbf{n}_{i} \beta_{i}$ for $\mathbf{n}=\left(\mathbf{n}_{i}\right) \in \mathbb{Z}^{m}$. Set $\widetilde{\Lambda}=\left\{\mathbf{n} \in \mathbb{Z}_{\geq 0}^{m} \mid f(\mathbf{n}) \in 2 \mathcal{P}\right\}$. We write $A^{(j)}=\sum_{\mathbf{n} \in \widetilde{\Lambda}} A_{\mathbf{n}}^{(j)} E^{\mathbf{n}}$ where $A_{\mathbf{n}}^{(j)} \in$ $M(r, 1, \mathbb{C})$. Let $\alpha \in \Sigma^{+}$and $E_{\alpha} \in \mathfrak{g}_{\alpha}$. Since $\mathfrak{k} u_{\lambda}=0$, we have $\left(\theta\left(E_{\alpha}\right)+E_{\alpha}\right) u_{\lambda}=$ 0 . Hence $\left(\theta\left(E_{\alpha}\right)+E_{\alpha}\right) \sum_{j} \sum_{\mathbf{n}} A_{\mathbf{n}}^{(j)} E^{\mathbf{n}} v_{j}=0$.

By applying Corollary 3.9 we have

$$
\sum_{j=1}^{r}\left(\sum_{\mathbf{n} \in \widetilde{\Lambda}} A_{\mathbf{n}}^{(j)}\left(\theta\left(E_{\alpha}\right)+E_{\alpha}\right) E^{\mathbf{n}}\right)^{\left(w_{i} \lambda-w_{j} \lambda-\alpha\right)} v_{j}=0
$$

for $i=1,2, \ldots, r$. On one hand, if $w_{i} \lambda-w_{j} \lambda \notin 2 \mathcal{P}_{+}$, then

$$
\left(\sum_{\mathbf{n} \in \widetilde{\Lambda}} A_{\mathbf{n}}^{(j)}\left(\theta\left(E_{\alpha}\right)+E_{\alpha}\right) E^{\mathbf{n}}\right)^{\left(w_{i} \lambda-w_{j} \lambda-\alpha\right)}=0 .
$$

On the other hand,

$$
\left(\sum_{\mathbf{n} \in \widetilde{\Lambda}} A_{\mathbf{n}}^{(i)}\left(\theta\left(E_{\alpha}\right)+E_{\alpha}\right) E^{\mathbf{n}}\right)^{(-\alpha)}=A_{\mathbf{0}}^{(i)} \theta\left(E_{\alpha}\right) .
$$

Hence we have

$$
A_{\mathbf{0}}^{(i)} \theta\left(E_{\alpha}\right) v_{i} \in \sum_{j \in \mathcal{W}(i)} U(\mathfrak{g}) v_{j} .
$$

Since $A_{\mathbf{0}}^{(i)}=\overline{A^{(i)}} \neq 0$, we have

$$
\theta\left(E_{\alpha}\right) v_{i} \in \sum_{j \in \mathcal{W}(i)} U(\mathfrak{g}) v_{j} .
$$

Next let $X$ be an element of $\mathfrak{m}$. By Corollary 3.9, we have

$$
\sum_{j=1}^{r}\left(\sum_{\mathbf{n} \in \widetilde{\Lambda}} A_{\mathbf{n}}^{(j)} X E^{\mathbf{n}}\right)^{\left(w_{i} \lambda-w_{j} \lambda\right)} v_{j}=0 .
$$

We can prove $X v_{i} \in \sum_{j \in \mathcal{W}(i)} U(\mathfrak{g}) v_{j}$ by the same argument. 
Corollary 5.3. $\quad$ Put $V(\lambda)=\sum_{i} U(\mathfrak{g}) v_{i} \subset \widehat{\mathcal{E}}(\mathfrak{g}, \mathfrak{n}) \otimes_{U(\mathfrak{g})} I(\lambda)$. Then we have $V(\lambda)=J(I(\lambda))$.

Proof. By Theorem 4.1, we have $u_{\lambda}=A v_{\lambda}$. Since $I(\lambda)$ is generated by $u_{\lambda}$, the module $\widehat{\mathcal{E}}(\mathfrak{g}, \mathfrak{n}) \otimes_{U(\mathfrak{g})} I(\lambda)$ is generated by $v_{1}, \ldots, v_{r}$ as an $\widehat{\mathcal{E}}(\mathfrak{g}, \mathfrak{n})$-module.

By Proposition 5.1, $V(\lambda)$ is finitely generated as a $U(\mathfrak{n})$-module. By applying Proposition 3.3, we see that the map $\widehat{\mathcal{E}}(\mathfrak{g}, \mathfrak{n}) \otimes_{U(\mathfrak{g})} V(\lambda) \rightarrow \prod_{\mu \in \mathfrak{a}^{*}} V(\lambda)_{\mu}$ is bijective. Hence $\widehat{\mathcal{E}}(\mathfrak{g}, \mathfrak{n}) \otimes_{U(\mathfrak{g})} V(\lambda) \rightarrow \widehat{\mathcal{E}}(\mathfrak{g}, \mathfrak{n}) \otimes_{U(\mathfrak{g})} I(\lambda)$ is injective by Proposition 3.8. This map is also surjective since $v_{1}, v_{2}, \ldots, v_{r}$ are generators of $\widehat{\mathcal{E}}(\mathfrak{g}, \mathfrak{n}) \otimes_{U(\mathfrak{g})} I(\lambda)$.

We have $\widehat{\mathcal{E}}(\mathfrak{g}, \mathfrak{n}) \otimes_{U(\mathfrak{g})} V(\lambda)=\widehat{\mathcal{E}}(\mathfrak{g}, \mathfrak{n}) \otimes_{U(\mathfrak{g})} I(\lambda)$. Since $I(\lambda)$ and $V(\lambda)$ are finitely generated as $U(\mathfrak{n})$-modules, we have

$$
\begin{aligned}
\widehat{\mathcal{E}}(\mathfrak{g}, \mathfrak{n}) \otimes_{U(\mathfrak{g})} I(\lambda) & =\widehat{J}(I(\lambda)), \\
\widehat{\mathcal{E}}(\mathfrak{g}, \mathfrak{n}) \otimes_{U(\mathfrak{g})} V(\lambda) & =\widehat{J}(V(\lambda)),
\end{aligned}
$$

by Proposition 3.1. Hence we have $J(I(\lambda))=J(V(\lambda))=V(\lambda)$ by Corollary 3.5.

Recall the definition of generalized Verma modules. Set $\overline{\mathfrak{p}}=\theta(\mathfrak{p})$ and $\overline{\mathfrak{n}}=\theta(\mathfrak{n})$.

Definition 5.4 (Generalized Verma module). Let $\mu \in \mathfrak{a}^{*}$. Define the one-dimensional representation $\mathbb{C}_{\rho+\mu}$ of $\overline{\mathfrak{p}}$ by $(X+Y+Z) v=(\rho+\mu)(Y) v$ for $X \in \mathfrak{m}, Y \in \mathfrak{a}, Z \in \overline{\mathfrak{n}}, v \in \mathbb{C}_{\rho+\mu}$. We define a $U(\mathfrak{g})$-module $M(\mu)$ by

$$
M(\mu)=U(\mathfrak{g}) \otimes_{U(\overline{\mathfrak{p}})} \mathbb{C}_{\rho+\mu} .
$$

This is called the generalized Verma module.

Set $V_{i}=\sum_{j>i} U(\mathfrak{g}) v_{j}$. By the universality of tensor products, any $U(\overline{\mathfrak{p}})$ module homomorphism $\mathbb{C}_{\rho+\mu} \rightarrow V$ can be uniquely extended to the $U(\mathfrak{g})$ module homomorphism $M(\mu) \rightarrow V$ for a $U(\mathfrak{g})$-module $V$. In particular, we have a surjective $U(\mathfrak{g})$-module homomorphism $M\left(w_{i} \lambda\right) \rightarrow V_{i} / V_{i+1}$. We shall show that $V_{i} / V_{i+1}$ is isomorphic to the generalized Verma module using the theory of characters.

Let $G$ be a connected Lie group such that $\operatorname{Lie}(G)=\mathfrak{g}_{0}, K$ its maximal compact subgroup with its Lie algebra $\mathfrak{k}_{0}, P$ the parabolic subgroup whose Lie algebra is $\mathfrak{p}_{0}$ and $P=M A N$ the Langlands decomposition of $P$ where Lie algebra of $M$ (resp. $A, N)$ is $\mathfrak{m}_{0}\left(\right.$ resp. $\left.\mathfrak{a}_{0}, \mathfrak{n}_{0}\right)$. 
Since $I(w \lambda)=I(\lambda)$ for $w \in W$, we may assume that $\operatorname{Re} \lambda$ is dominant, i.e., $\operatorname{Re} \lambda\left(H_{i}\right) \geq 0$ for each $i=1,2, \ldots, l$. By a result of Kostant [Kos75, Theorem 2.10.3], $I(\lambda)$ is isomorphic to the space of $K$-finite vectors of the non-unitary principal series representation $\operatorname{Ind}_{P}^{G}(1 \otimes \lambda)$. The character of this representation is calculated by Harish-Chandra (See Knapp [Kna01, Proposition 10.18]). Before we state it, we fix some notation. Let $H=T A$ be a maximally split Cartan subgroup, $\mathfrak{h}_{0}$ its Lie algebra, $T=H \cap M, \Delta$ the root system of $H, \Delta^{+}$the positive system compatible with $\Sigma^{+}, \Delta_{I}$ the set of imaginary roots, $\Delta_{I}^{+}=\Delta^{+} \cap \Delta_{I}$ and $\xi_{\alpha}$ the one-dimensional representation of $H$ whose derivation is $\alpha$ for $\alpha \in \mathfrak{h}^{*}$. Under these notation, the distribution character $\Theta_{G}(I(\lambda))$ of $I(\lambda)$ is as follows;

$$
\Theta_{G}(I(\lambda))(t a)=\frac{\sum_{w \in W} \xi_{\rho+w \lambda}(a)}{\prod_{\alpha \in \Delta^{+} \backslash \Delta_{I}^{+}}\left|1-\xi_{\alpha}(t a)\right|} \quad(t \in T, a \in A) .
$$

We will use the Osborne conjecture, which was proved by Hecht and Schmid [HS83a, Theorem 3.6]. To state it, we must define the character of $J(\mathbb{X})$ for a Harish-Chandra module $\mathbb{X}$. Recall that $J(\mathbb{X})$ is an object of the category $\mathcal{O}_{P}^{\prime}$, i.e.,

(1) the actions of $M \cap K$ and $\mathfrak{g}$ are compatible,

(2) $J(\mathbb{X})$ splits under $\mathfrak{a}$ into the direct sum of generalized weight spaces, each of them being a Harish-Chandra module for $M A$,

(3) $J(\mathbb{X})$ is $U(\overline{\mathfrak{n}})$ - and $Z(\mathfrak{g})$-finite

(See Hecht and Schmid [HS83b, (34)Lemma]). For an object $V$ of $\mathcal{O}_{P}^{\prime}$, we define the character $\Theta_{P}(V)$ of $V$ by

$$
\Theta_{P}(V)=\sum_{\mu \in \mathfrak{a}^{*}} \Theta_{M A}\left(V_{\mu}\right),
$$

where $V_{\mu}$ is the generalized $\mu$-weight space of $V$. Let $G^{\prime}$ be the set of regular elements of $G$. Set

$$
A^{-}=\left\{a \in A \mid \alpha(\log a)<0 \text { for all } \alpha \in \Sigma^{+}\right\},
$$

$$
\begin{aligned}
& (M A)^{-}= \\
& \text {interior of }\left\{g \in M A \mid \prod_{\alpha \in \Delta^{+} \backslash \Delta_{I}^{+}}\left(1-\xi_{\alpha}(g a)\right) \geq 0 \text { for all } a \in A^{-}\right\} \text {in } M A .
\end{aligned}
$$


Then the Osborn conjecture says that $\Theta_{G}(\mathbb{X})$ and $\Theta_{P}(J(\mathbb{X}))$ coincide on $(M A)^{-} \cap G^{\prime}$ (See Hecht and Schmid [HS83b, (42)Lemma]). It is easy to calculate the character of a generalized Verma module. We have

$$
\Theta_{P}(M(\mu))(t a)=\frac{\xi_{\rho+\mu}(a)}{\prod_{\alpha \in \Delta^{+} \backslash \Delta_{I}^{+}}\left(1-\xi_{\alpha}(t a)\right)} \quad(t \in T, a \in A) .
$$

Consequently we have

$$
\Theta_{P}(J(I(\lambda)))=\sum_{w \in W} \Theta_{P}(M(w \lambda)) .
$$

This implies the following theorem when $\lambda$ is regular.

Theorem 5.5. $\quad$ There exists a filtration $0=V_{r+1} \subset V_{r} \subset \cdots \subset V_{1}=$ $J(I(\lambda))$ of $J(I(\lambda))$ such that $V_{i} / V_{i+1}$ is isomorphic to $M\left(w_{i} \lambda\right)$ for an arbitrary $\lambda \in \mathfrak{a}^{*}$. Moreover if $w \lambda-\lambda \notin 2 \mathcal{P}$ for all $w \in W \backslash\{e\}$ then $J(I(\lambda)) \simeq$ $\bigoplus_{w \in W} M(w \lambda)$.

Proof. The first part of the theorem has already been proved. For the second part, consider the exact sequence

$$
0 \longrightarrow V_{i+1} \longrightarrow V_{i} \longrightarrow M\left(w_{i} \lambda\right) \longrightarrow 0 .
$$

If $w \lambda-\lambda \notin 2 \mathcal{P}$ for all $w \in W \backslash\{e\}$, then $v_{i} \in V_{i}$ is a lowest weight vector from Proposition 5.1. Hence we have a map $M\left(w_{i} \lambda\right) \rightarrow V_{i}$, which gives a splitting of the above exact sequence.

\section{$\S 6 . \quad$ Structure of Jacquet Modules (Singular Case)}

In this section, we shall prove Theorem 5.5 in the singular case using the translation principle. We keep the notation of Section 5 . Let $\lambda^{\prime}$ be an element of $\mathfrak{a}^{*}$ such that the following conditions hold:

- The weight $\lambda^{\prime}$ is regular.

- The weight $\left(\lambda-\lambda^{\prime}\right) / 2$ is integral.

- The real part of $\lambda^{\prime}$ belongs to the same Weyl chamber that the real part of $\lambda$ belongs to.

First we define the translation functor $T_{\lambda^{\prime}}^{\lambda}$. Let $\mathbb{X}$ be a $U(\mathfrak{g})$-module which has an infinitesimal character $\lambda^{\prime}$. (We regard $\mathfrak{a}^{*} \subset \mathfrak{h}^{*}$.) We define $T_{\lambda^{\prime}}^{\lambda}(\mathbb{X})$ by $T_{\lambda^{\prime}}^{\lambda}(\mathbb{X})=P_{\lambda}\left(\mathbb{X} \otimes E_{\lambda-\lambda^{\prime}}\right)$ where: 
- $E_{\lambda-\lambda^{\prime}}$ is the finite-dimensional irreducible representation of $\mathfrak{g}$ with an extreme weight $\lambda-\lambda^{\prime}$.

- $P_{\lambda}(V)=\left\{v \in V \mid\right.$ for some $n>0$ and all $\left.z \in Z(\mathfrak{g}),(z-\lambda(\widetilde{\chi}(z)))^{n} v=0\right\}$ where $Z(\mathfrak{g})$ is the center of $U(\mathfrak{g})$ and $\tilde{\chi}: Z(\mathfrak{g}) \rightarrow U(\mathfrak{h})$ is the Harish-Chandra homomorphism.

Notice that $P_{\lambda}$ and $T_{\lambda^{\prime}}^{\lambda}$ are exact functors. Since the functors $J$ and $T_{\lambda^{\prime}}^{\lambda}$ commute [Mat90, Proposition 3.2.1], Theorem 5.5 in the singular case follows from the following two identities.

(1) $T_{\lambda^{\prime}}^{\lambda}\left(I\left(\lambda^{\prime}\right)\right)=I(\lambda)$.

(2) $T_{\lambda^{\prime}}^{\lambda}\left(M\left(w \lambda^{\prime}\right)\right)=M(w \lambda)$.

The following lemma is important to prove these identities.

Lemma 6.1. Let $\nu$ be a weight of $E_{\lambda-\lambda^{\prime}}$ and $w \in W$. Assume $\nu=$ $w \lambda-\lambda^{\prime}$. Then $\nu=\lambda-\lambda^{\prime}$.

Proof. See Vogan [Vog81, Lemma 7.2.18].

Proof of $T_{\lambda^{\prime}}^{\lambda}\left(I\left(\lambda^{\prime}\right)\right)=I(\lambda)$. We may assume that $\lambda^{\prime}$ is dominant. Notice that we have $I\left(\lambda^{\prime}\right) \simeq \operatorname{Ind}_{P}^{G}\left(1 \otimes \lambda^{\prime}\right)_{K}$. Let $0=E_{0} \subset E_{1} \subset E_{2} \subset \cdots \subset E_{n}=$ $E_{\lambda-\lambda^{\prime}}$ be a $P$-stable filtration with the trivial induced action of $N$ on $E_{i} / E_{i-1}$. We may assume that $E_{i} / E_{i-1}$ is irreducible. Let $\nu_{i}$ be the highest weight of $E_{i} / E_{i-1}$. Notice that $\operatorname{Ind}_{P}^{G}\left(1 \otimes \lambda^{\prime}\right) \otimes E_{\lambda-\lambda^{\prime}}=\operatorname{Ind}_{P}^{G}\left(\left(1 \otimes \lambda^{\prime}\right) \otimes E_{\lambda-\lambda^{\prime}}\right)$. Then $\operatorname{Ind}_{P}^{G}\left(1 \otimes \lambda^{\prime}\right) \otimes E_{\lambda-\lambda^{\prime}}$ has a filtration $\left\{M_{i}\right\}$ such that $M_{i} / M_{i-1} \simeq \operatorname{Ind}_{P}^{G}\left(\left(1 \otimes \lambda^{\prime}\right) \otimes\right.$ $\left.\left(E_{i} / E_{i-1}\right)\right)$. Since $\operatorname{Ind}_{P}^{G}\left(\left(1 \otimes \lambda^{\prime}\right) \otimes\left(E_{i} / E_{i-1}\right)\right)$ has an infinitesimal character $\lambda+\nu_{i}, P_{\lambda}\left(M_{i} / M_{i-1}\right)=0$ if $\nu_{i} \neq w \lambda-\lambda^{\prime}$ for all $w \in W$. By Lemma 6.1 we have $T_{\lambda^{\prime}}^{\lambda}\left(\operatorname{Ind}_{P}^{G}\left(1 \otimes \lambda^{\prime}\right)\right)=\operatorname{Ind}_{P}^{G}\left(\left(1 \otimes \lambda^{\prime}\right) \otimes\left(E_{i} / E_{i-1}\right)\right)$ where $\nu_{i}=\lambda-\lambda^{\prime}$. By the conditions of $\lambda^{\prime}$, the action of $M$ on the $\left(\lambda-\lambda^{\prime}\right)$-weight space of $E_{\lambda-\lambda^{\prime}}$ is trivial. Consequently $T_{\lambda^{\prime}}^{\lambda}\left(\operatorname{Ind}_{P}^{G}\left(1 \otimes \lambda^{\prime}\right)\right)=\operatorname{Ind}_{P}^{G}\left(\left(1 \otimes \lambda^{\prime}\right) \otimes\left(\lambda-\lambda^{\prime}\right)\right)=\operatorname{Ind}_{P}^{G}(1 \otimes \lambda)$.

Proof of $T_{\lambda^{\prime}}^{\lambda}\left(M\left(w \lambda^{\prime}\right)\right)=M(w \lambda)$. We may assume $w=e \in W$. Since $M\left(\lambda^{\prime}\right) \otimes E_{\lambda-\lambda^{\prime}}=U(\mathfrak{g}) \otimes\left(\mathbb{C}_{\lambda^{\prime}} \otimes E_{\lambda-\lambda^{\prime}}\right)$, the equation follows by the same argument of the proof of $T_{\lambda^{\prime}}^{\lambda}\left(I\left(\lambda^{\prime}\right)\right)=I(\lambda)$.

\section{Acknowledgments}

The author is grateful to his advisor Hisayosi Matumoto for his advice and support. He would also like to thank Professor Toshio Oshima for his comments. 


\section{References}

[Cas80] W. Casselman, Jacquet modules for real reductive groups, in Proceedings of the International Congress of Mathematicians (Helsinki, 1978), 557-563, Acad. Sci. Fennica, Helsinki.

[CO78] W. Casselman and M. S. Osborne, The restriction of admissible representations to $\mathfrak{n}$, Math. Ann. 233 (1978), no. 3, 193-198.

[Col85] D. H. Collingwood, Representations of rank one Lie groups, Pitman, Boston, MA, 1985.

[Col91] _ Jacquet modules for semisimple Lie groups having Verma module filtrations, J. Algebra 136 (1991), no. 2, 353-375.

[GW80] R. Goodman and N. R. Wallach, Whittaker vectors and conical vectors, J. Funct. Anal. 39 (1980), no. 2, 199-279.

[Hel62] S. Helgason, Differential geometry and symmetric spaces, Academic Press, New York, 1962.

[HS83a] H. Hecht and W. Schmid, Characters, asymptotics and n-homology of HarishChandra modules, Acta Math. 151 (1983), no. 1-2, 49-151.

[HS83b] _ On the asymptotics of Harish-Chandra modules, J. Reine Angew. Math. 343 (1983), 169-183.

$\left[\mathrm{KKM}^{+} 78\right]$ M. Kashiwara, A. Kowata, K. Minemura, K. Okamoto, T. Oshima and M. Tanaka, Eigenfunctions of invariant differential operators on a symmetric space, Ann. of Math. (2) 107 (1978), no. 1, 1-39.

[Kna01] A. W. Knapp, Representation theory of semisimple groups, Reprint of the 1986 original, Princeton Univ. Press, Princeton, NJ, 2001.

[Kna02] _ Lie groups beyond an introduction, Second edition, Birkhäuser Boston, Boston, MA, 2002.

[Kos75] B. Kostant, On the existence and irreducibility of certain series of representations, in Lie groups and their representations (Proc. Summer School, Bolyai János Math. Soc., Budapest, 1971), 231-329, Halsted, New York.

[Kos78] _ On Whittaker vectors and representation theory, Invent. Math. 48 (1978), no. 2, 101-184.

[Lep75] J. Lepowsky, Existence of conical vectors in induced modules, Ann. of Math. (2) 102 (1975), no. 1, 17-40.

[Mat90] H. Matumoto, Whittaker modules associated with highest weight modules, Duke Math. J. 60 (1990), no. 1, 59-113.

[Osh84] T. Ōshima, Boundary value problems for systems of linear partial differential equations with regular singularities, in Group representations and systems of differential equations (Tokyo, 1982), 391-432, Adv. Stud. Pure Math., 4, NorthHolland, Amsterdam.

[Osh88] _ A realization of semisimple symmetric spaces and construction of boundary value maps, in Representations of Lie groups, Kyoto, Hiroshima, 1986, 603650, Adv. Stud. Pure Math., 14, Academic Press, Boston, MA.

[SW82] J. T. Stafford and N. R. Wallach, The restriction of admissible modules to parabolic subalgebras, Trans. Amer. Math. Soc. 272 (1982), no. 1, 333-350.

[Vog81] D. A. Vogan, Jr., Representations of real reductive Lie groups, Progress in Mathematics, vol. 15, Birkhäuser Boston, Mass., 1981.

[Wal88] N. R. Wallach, Real reductive groups. I, Academic Press, Boston, MA, 1988. 